\title{
Homogenization of Continuum-Scale Transport Properties from Molecular Dynamics Simulations: An Application to Aqueous-Phase Methane Diffusion in Silicate Channels
}

\author{
Tom Pace ${ }^{1, *}$, Hadi Rahmaninejad ${ }^{2, *}$, Bin Sun $^{1}$, and Peter \\ Kekenes-Huskey ${ }^{1}$ \\ ${ }^{1}$ Department of Cell \& Molecular Physiology, Loyola University \\ Chicago \\ ${ }^{2}$ Department of Physics, Virginia Tech \\ ${ }^{*}$ Corresponding authors
}

July 26, 2021 


\section{Abstract}

Silica-based materials including zeolites are commonly used for wide ranging applications including separations and catalysis. Substrate transport rates in these materials can significantly influence the efficiency of such applications. Two factors that contribute to transport rates include 1) the porosity of the silicate matrix and 2) non-bonding interactions between the diffusing species and the silicate surface. These contributions generally emerge from disparate length scales, namely 'microscopic' (roughly nanometer-scale) and 'macroscopic' (roughly micron-scale), respectively. Here, we develop a simulation framework to estimate the simultaneous impact of these factors on methane mass transport in silicate channels. Specifically, we develop a model of methane transport using homogenization theory to obtain transport parameters valid at length scales of hundreds to thousands of nanometers. These parameters implicitly reflect interactions taking place at fractions of a nanometer. The inputs to the homogenization analysis are data from extensive molecular dynamics simulations that incorporate atomistic-scale interactions, processed to yield local diffusion coefficients and mean force potentials. With this model, we demonstrate how nuances in silicate hydration and silica/methane interactions impact methane diffusion rates in silicate materials, including the effects of silicate surface chemistry such as the presence of silanol groups. The molecular dynamics simulations indicate that methane diffusivity at the silica surface is lower than the bulk-like rates observed at the center of channels of sufficient width. However, potentials of mean force generally evidence attractive methane/silica interactions that enhance diffusion overall. By simultaneously accounting for both of these effects, we show that the effective diffusion coefficient for the nanoporous silicate can be approximately double the value of estimates assuming fully bulk-like behavior in the channel. This study therefore demonstrates the importance of determining diffusion coefficients and potentials of mean force at an atomistic level when estimating transport properties in bulk materials. Importantly, we provide a simple homogenization framework to incorporate these molecular-scale attributes into bulk material transport estimates. This hybrid homogenization/molecular dynamics approach will be of general use for describing small molecule transport in materials with detailed molecular interactions.

\section{Introduction}

Porous media are encountered in many applications in a variety of fields of science and engineering, including chemical reactors, groundwater analysis, petroleum extraction, and even some types of artificial organs [1, 2]. In particular, these applications often depend on the rate of transport of a given reactant, contaminant, or other chemical species through the fluid-filled pores of the material. The successful prediction of macroscopic transport properties such as Fickian diffusion coefficients requires proper consideration of the physical and chemical interactions within the system at smaller scales. Previous studies of the transport properties of porous media have noted that the structure and behavior of the pore fluid differs significantly from bulk conditions for pores that are sufficiently small. For example, recent molecular dynamics (MD) studies have shown that water under confinement exhibits changes in the dynamics of the hydrogen bond network of the water molecules, with effects similar to a reduction in temperature [3]. A study of water confined to a gap of 6.5 to $14.5 \AA$ between two graphene plates observed a layered water structure, with the number of layers increasing as the gap widened [4]. Water confined to metal-organic frameworks has been found to exhibit spatially heterogenous water structure [5]. 
Other studies, described below, have used various parameters to quantify the difference in fluid transport properties between bulk and confined conditions. Such approaches include spatial variations of the diffusion coefficient within the pore space, or the consideration of a Potential of Mean Force (PMF) within the pore space.

A variety of prior studies have investigated hydrocarbon transport in silicate materials. Hansen et al. [6] and Hansen and Keil [7] considered the alkylation of benzene within a zeolite catalyst. The reaction and diffusion were considered in the gas phase. Consequently, the Maxwell-Stefan diffusion equations were used rather than Fickian or Smoluchowski diffusion. Information from MD and kinetic Monte Carlo simulations was used to provide inputs to the continuum diffusion model. The continuum diffusion model was evaluated analytically under an assumption of spherical symmetry, rather than a numerical simulation using the pore geometry. One key focus of the study was to predict reaction rates agreeing with experiment, which is not attempted herein.

In an analysis conducted by Bui et al. [8], a channel of one nanometer in width was studied in various materials, including silica. The study computed the diffusion coefficient within the channel using the Mean Squared Displacement (MSD) of unrestrained methane gas molecules. This results in a coefficient value that does not distinguish spatial variations within the channel, reducing the accuracy in inhomogeneous situations [9]. No multi-scaling method was applied to the result; behavior at larger scales was not investigated. In addition to using a metadynamics approach to evaluate the free energy variations within the channel, umbrella sampling was also applied. Finally, the silica structure of the Bui et al. [8] study does not appear to include silanol terminations at the surface. The silanol groups present on the silicate surfaces studied herein would be expected to increase the affinity for water molecules, leading to different results for the water density within the channel, and consequently affecting the PMF and diffusion coefficients. The study found anisotropy in the diffusion coefficient, with differing coefficients for directions perpendicular to the silica face than parallel to it.

Collin et al. [10] used MD to study water in a cylindrical nanopore of $1 \mathrm{~nm}$ diameter. In these simulations, $0-25 \%$ of the silanol groups on the pore surface were deprotonated, leaving behind a negative surface charge that was found to strongly affect the water density near the surface. A variety of alkali metal counterions were used to balance the net electrical charge of the system, but the results were not found to vary significantly among the different alkali metals. No PMF calculation was included in the study. The MSD of unrestrained water molecules was used to obtain the self-diffusion coefficient of water. The authors note that this procedure had lower precision in the nanochannel than in bulk water. While this lower precision was not investigated further, we speculate that it might have been caused by inhomogeneity of the conditions within the pore, insufficient sampling in thermodynamically unfavorable regions, or the influence of a PMF. A diffusion coefficient for the entire pore was also calculated by analysis of the diffusion behavior of a subset of water molecules. The authors found agreement between these two procedures.

Here, we propose a multi-scale model incorporating the chemical and physical processes that dominate different portions of the hierarchy of scales. We demonstrate these techniques on an example system consisting of methane as the solute, water as the solvent, and a nanoporous silicate medium. The silicate geometry is shown in Figure 1. The mathematical procedure of homogenization $[11,12]$ is used to aggregate the non-bulk behavior within the nanopore into an effective diffusion coefficient for a larger analysis scale. In order to include spatial variations of the local diffusion coefficient and the presence of a PMF, the homogenization procedure is based on the Smoluchowski diffusion equation. 
Along with the local diffusion coefficent and the PMF, local water density values are also used to assess the change of the water structure from bulk conditions. These parameters are measured inside the pore from MD simulation trajectories. A similar approach has previously been applied to the permeability, diffusion, and consolidation properties of a bentonite clay [13].

We explored variations of the nanoporous silicate system including alterations of the porosity and changes to the surface chemistry of the silicate. We found that the local variations in the diffusion coefficient, alone, tend to result in suppressed diffusion within the pore overall. In the fully protonated case, the channel contains areas where the PMF is attractive to methane relative to bulk water, and the effect of this potential is to result in a net increase in the effective diffusion coefficient. However, partial deprotonation of the silicate surface appears to remove the attractive regions of the PMF, resulting in a further reduction of the effective diffusion coefficient. Such deprotonation would be present under most $\mathrm{pH}$ conditions [10]. 
a)

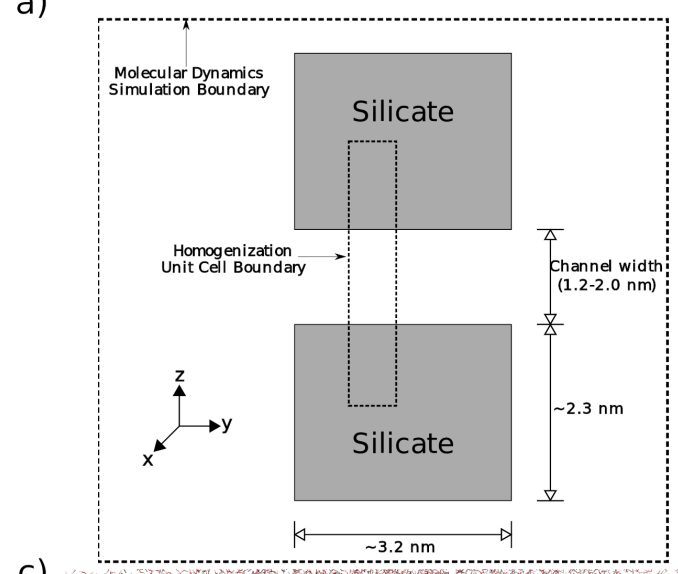

c)

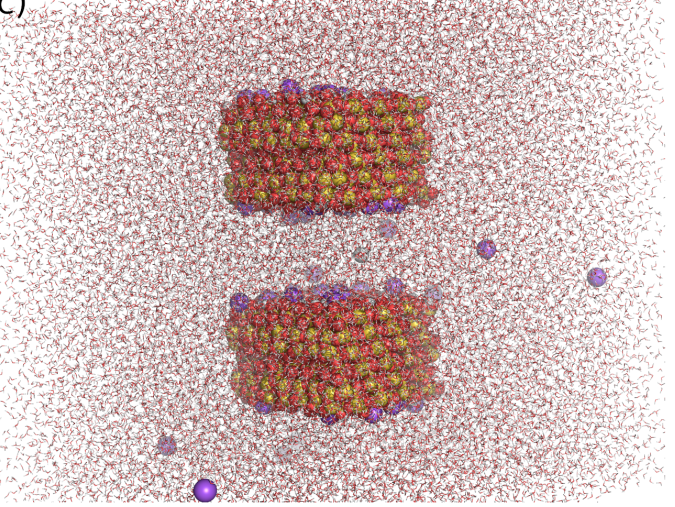

b)
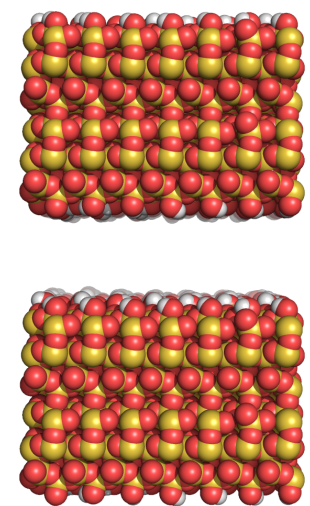

d)

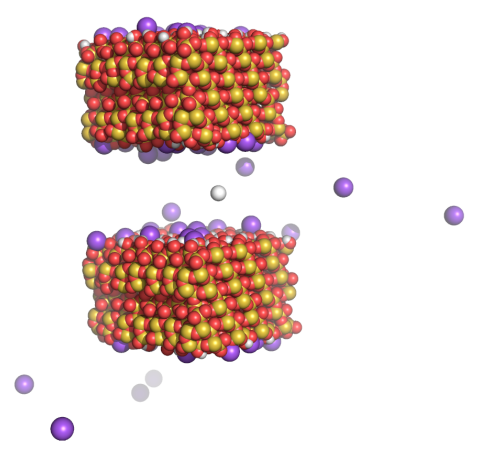

Figure 1: Silicon dioxide $\left(\mathrm{SiO}_{2}\right)$ matrix with 'slit' of variable height (1.2-2.0 $\mathrm{nm}$ ). A periodic material with this geometry would contain multiple parallel channels. a) Sketch showing key dimensions, coordinate axes, and simulation boundaries. b) Rendering of the $1.2 \mathrm{~nm}$ channel along the $\mathrm{X}$-axis in the fully protonated condition. Silicon $(\mathrm{Si})$ atoms are tan, oxygen $(\mathrm{O})$ atoms are red, and hydrogen $(\mathrm{H})$ atoms are white. c) Oblique rendering of the $2.0 \mathrm{~nm}$ channel with $27 \%$ deprotonation after solvation and equilibration. Sodium $(\mathrm{Na})$ ions are shown as purple spheres. d) Same view as (c) without water molecules. The methane molecule is visible in white, near mid-channel. The silicate structures provided in Emami et al. [14, 15] were used to construct this geometry in the MD simulations. 


\section{Results}

We conducted a series of homogenization and MD simulations to assess how methane diffusion at larger scales is influenced by non-bulk behavior within the nano-scale silicate channel.

First, we applied the homogenization analysis to the silicate channel geometry under the assumption that the diffusion behavior of methane through water within the channel would be identical to the diffusion behavior of methane in bulk water. Under this assumption, the effective diffusion coefficient for the nanoporous silicate can also be obtained by other calculation procedures. Comparison of the results from different methods also serves to validate the homogenization procedure. These simulations are discussed in Sect. 3.1. A further validation of the homogenization procedures, using an assumed potential within the channel, is discussed in Sect. 3.2.

Next, we used MD simulations to quantify the differences between aqueous methane diffusion in the silicate channel and the diffusion of methane in bulk water. This included bulk simulations described in Sect. 3.3 for validation and comparison purposes. Sect. 3.4 then describes the findings from the MD simulations for an example channel. Variations of the channel size, and thus the porosity of the nanporous silicate, are examined in Sect. 3.5, and variations in the degree of protonation of the channel surfaces are examined in Sect. 3.6.

Finally, the transport behavior observed in the MD simulations is incorporated into the homogenization simulations in Sect. 3.7.

\subsection{Homogenization assuming bulk behavior in the chan- nel}

Solvated in bulk water, a single methane molecule experiences no net PMF, and the diffusion coefficient is both isotropic and homogenous. Assuming such conditions inside the silicate channel leads to a model with purely Fickian diffusion. The effective diffusion coefficient for larger scales can be estimated from Equation 1 [2].

$$
\frac{D_{\text {macro }}}{D_{\text {bulk }}}=\frac{\epsilon \delta}{\tau}
$$

where

$$
\begin{aligned}
& \epsilon=\text { effective porosity, } \leq 1 \\
& \delta=\text { constrictivity, } \leq 1 \\
& \tau=\text { tortuosity, } \geq 1
\end{aligned}
$$

In this case, the pore is straight, giving a tortuosity of $\tau=1$. The assumption of bulk conditions also implies a constrictivity of $\delta=1$. The effective porosity, $\epsilon$, is easily computed from the ratio of the channel cross-sectional area to the unit cell area. For the channel geometry shown in Figure 1, this effective porosity is identical to the porosity of the silicate material. The porosity, $\phi$, is the ratio of the channel volume to the unit cell volume, and is also known as the free volume fraction for this reason.

Equation 1 provides an analytical estimate of the effective diffusion coefficient that can be compared to the results from homogenization simulations. However, the parameters of Equation 1 are not easily obtained in more complicated situations.

Homogenization simulations were conducted for three different channel widths. The resulting effective diffusion coefficients are shown in Table 1. These results are also plotted in the left panel of Figure 9. For further validation, the effective diffusion coefficient was also computed directly from the fluxes obtained by simulations of Fick's Law as described in Sect. 6.2.3. All three methods gave the same results to at least three significant figures. 
Table 1: Effective diffusion coefficient assuming bulk behavior in the channel. The results are normalized to the bulk diffusion coefficient.

\begin{tabular}{cccc}
$\begin{array}{c}\text { Channel Width } \\
{[\mathrm{nm}]}\end{array}$ & $\begin{array}{c}\text { Analytical } \\
\text { Estimate }\end{array}$ & $\begin{array}{c}\text { Homogenization } \\
\text { Result }\end{array}$ & $\begin{array}{c}\text { Flux-based } \\
\text { Result }\end{array}$ \\
\hline 1.2 & 0.207 & 0.207 & 0.207 \\
1.6 & 0.258 & 0.258 & 0.258 \\
2.0 & 0.303 & 0.303 & 0.303
\end{tabular}

\subsection{Homogenization assuming a quadratic potential in the channel}

The analysis in Sect. 3.1 validates the homogenization approach for case of purely Fickian diffusion, where there is no mean force potential driving or retarding the diffusion. Situations where there is a spatially varying potential are described by the Smoluchowski equation. To validate the homogenization simulation methodology for the Smoluchowski diffusion equation, we conducted additional simulations that could be compared to a theoretical result. The local diffusion coefficient was assumed to be isotropic and equal to the bulk diffusion coefficient at all locations within the channel. A quadratic potential, as described by Equation 2 was also applied.

$$
V(z)=A \bar{z}^{2}+B
$$

In this quadratic potential, $\bar{z}$ is the distance in the $z$-coordinate from the center of the channel. The values of the constants $A$ and $B$ were selected such that the minimum and maximum values of the potential within the channel were in the same range as the minima and maxima of the PMF results from the MD simulations, which we present later in Sect. 3.5. This required the potential to rise more quickly in smaller channels than in wider ones, which is a phenomenon also observed in the PMF data from the MD simulations. These quadratic potentials are plotted in Figure S3. The analytical solution for this result is presented in the Supplement.

The effective diffusion coefficient results from these simulations are compared to both the analytical values and the results computed directly from fluxes obtained from simulations of the Smoluchowski equation in Table 2. All three methods gave the same results to at least three significant figures. The homogenization results and analytical calculation results are also plotted in the left panel of Figure 9.

Table 2: Effective diffusion coefficient for a quadratic potential and bulk diffusion coefficient. The results are normalized to the bulk diffusion coefficient.

\begin{tabular}{cccc}
$\begin{array}{c}\text { Channel Width } \\
{[\mathrm{nm}]}\end{array}$ & $\begin{array}{c}\text { Analytical } \\
\text { Estimate }\end{array}$ & $\begin{array}{c}\text { Homogenization } \\
\text { Result }\end{array}$ & $\begin{array}{c}\text { Flux-based } \\
\text { Result }\end{array}$ \\
\hline 1.2 & 0.289 & 0.289 & 0.289 \\
1.6 & 0.360 & 0.360 & 0.360 \\
2.0 & 0.423 & 0.423 & 0.423
\end{tabular}




\subsection{Behavior of methane in bulk water in molecular dy- namics simulations}

For later comparison to the MD simulation results within the silicate channel, and to validate our procedures, we performed simulations of a single methane molecule in bulk water. The diffusion coefficient of methane in bulk water was calculated from these simulations for comparison with experimental results presented in Witherspoon and Saraf [16].

First, a set of simulations was conducted where the methane molecule was not restrained. In this case, the MSD should increase linearly with respect to time, with a slope equal to $6 D$ [17]. Accordingly, for these simulations, the MSD was plotted against time and fit to a linear function, with the diffusion coefficient calculated from this fit. Results from three trials are shown in Figure S1. The resulting diffusion coefficients are shown in Table 3.

Table 3: Diffusion coefficient results from unrestrained methane simulations in bulk water.

\begin{tabular}{cc} 
Trial & $\begin{array}{c}\text { Diffusion } \\
\text { Coefficient } \\
\left(1 \times 10^{-5} \mathrm{~cm}^{2} / \mathrm{sec}\right)\end{array}$ \\
\hline Trial A & 2.39 \\
Trial B & 1.21 \\
Trial C & 4.51 \\
\hline Mean of Trials & \\
\pm Standard Error & $2.70 \pm 0.96$ \\
of the Mean &
\end{tabular}

While the use of unrestrained simulations for obtaining the local diffusion coefficient is relatively simple, it is not appropriate for situations where the diffusion coefficient exhibits significant spatial variation [9]. Accordingly, the local diffusion coefficient was also obtained from simulations of a restrained methane molecule in bulk water. The calculation procedure is described in Sect. 5.2 .

The restrained methane simulations in bulk water were conducted with two different force fields. The force field parameters for silicate from Emami et al. $[14,15]$ were desired for the simulations of the silicate channel. For compatibility with this force field, the force field parameters for the united atom approximation of methane were taken from Jorgensen, Madura, and Swenson [18], and the SPC water model was used. As this validation does not contain any silicate, the first force field used is a combination of the Jorgensen et al methane parameters and the SPC water model, designated as the Jorgensen/SPC force field. In contrast, Daldrop, Kowalik, and Netz [19] used the GROMOS 53A6 [20] force field in their validation, and found that it was capable of matching experimental results, so this force field was also tested for comparison to the Jorgensen/SPC force field. Each simulation was $16 \mathrm{~ns}$ in duration, and was conducted at a temperature of $298 \mathrm{~K}$. Both force fields gave MSD results that approximated the expected result of $k_{B} T / K$ (where $K$ is the spring constant for the harmonic restraining potential applied to the methane), and the two simulation values were in agreement with one another, as shown in Table S1.

An example Position Autocorrelation Function (PACF) curve from each simulation is shown in the upper panel of Figure 2. The diffusion coefficient for methane in bulk water was then calculated in both simulations from the PACF 
for each coordinate. The results are shown in Table 4. The GROMOS 53A6 force field matches the experimental results quite well, as reported by Daldrop, Kowalik, and Netz [19]. In contrast, the Jorgensen/SPC force field overestimates the diffusion coefficient by roughly $60 \%$, because it gives a slightly lower curve for the PACF as shown in the upper panel of Figure 2.

Table 4: Diffusion coefficient results from simulations of methane in bulk water. The error reported for the experimental result by Witherspoon and Saraf [16] was the standard deviation of the mean from four to six repeated runs of the experiment. The experimental result did not distinguish between different directions. The error for the molecular dynamics (MD) simulation results is the standard error of the mean over the three directions of measurement.

\begin{tabular}{cccc} 
Direction & $\begin{array}{c}\text { Experimental } \\
\text { Result [16] } \\
\left(1 \times 10^{-5} \mathrm{~cm}^{2} / \mathrm{sec}\right)\end{array}$ & $\begin{array}{c}\text { Jorgensen/SPC } \\
\text { Force Field } \\
\left(1 \times 10^{-5} \mathrm{~cm}^{2} / \mathrm{sec}\right)\end{array}$ & $\begin{array}{c}\text { GROMOS 53A6 } \\
\text { Force Field } \\
\left(1 \times 10^{-5} \mathrm{~cm}^{2} / \mathrm{sec}\right)\end{array}$ \\
\hline $\mathrm{x}$ & $\mathrm{n} / \mathrm{a}$ & 3.13 & 1.91 \\
$\mathrm{y}$ & $\mathrm{n} / \mathrm{a}$ & 2.96 & 1.73 \\
$\mathrm{z}$ & $\mathrm{n} / \mathrm{a}$ & 3.06 & 1.85 \\
\hline Mean Value & $1.88 \pm 0.01$ & $3.05 \pm 0.05$ & $1.83 \pm 0.05$
\end{tabular}

The lower panel of Figure 2 summarizes the validation results by averaging over the directional components. Notably, the MSD approach for unrestrained methane exhibited greater variation in the diffusion coefficient than the PACF approach for restrained methane. The PACF and MSD approaches using the Jorgensen/SPC force field gave notably higher diffusion coefficient values than the experimental results of Witherspoon and Saraf [16], but were in general agreement with one another. Accordingly, local diffusion coefficient values in the silicate channel computed using the Jorgensen/SPC force field should be normalized by the bulk value presented here for correct interpretation. Specifically, the local diffusion coefficient values are divided by $3.0 \times 10^{-5} \mathrm{~cm}^{2} / \mathrm{sec}$ to report the ratio of local diffusion to bulk diffusion. 

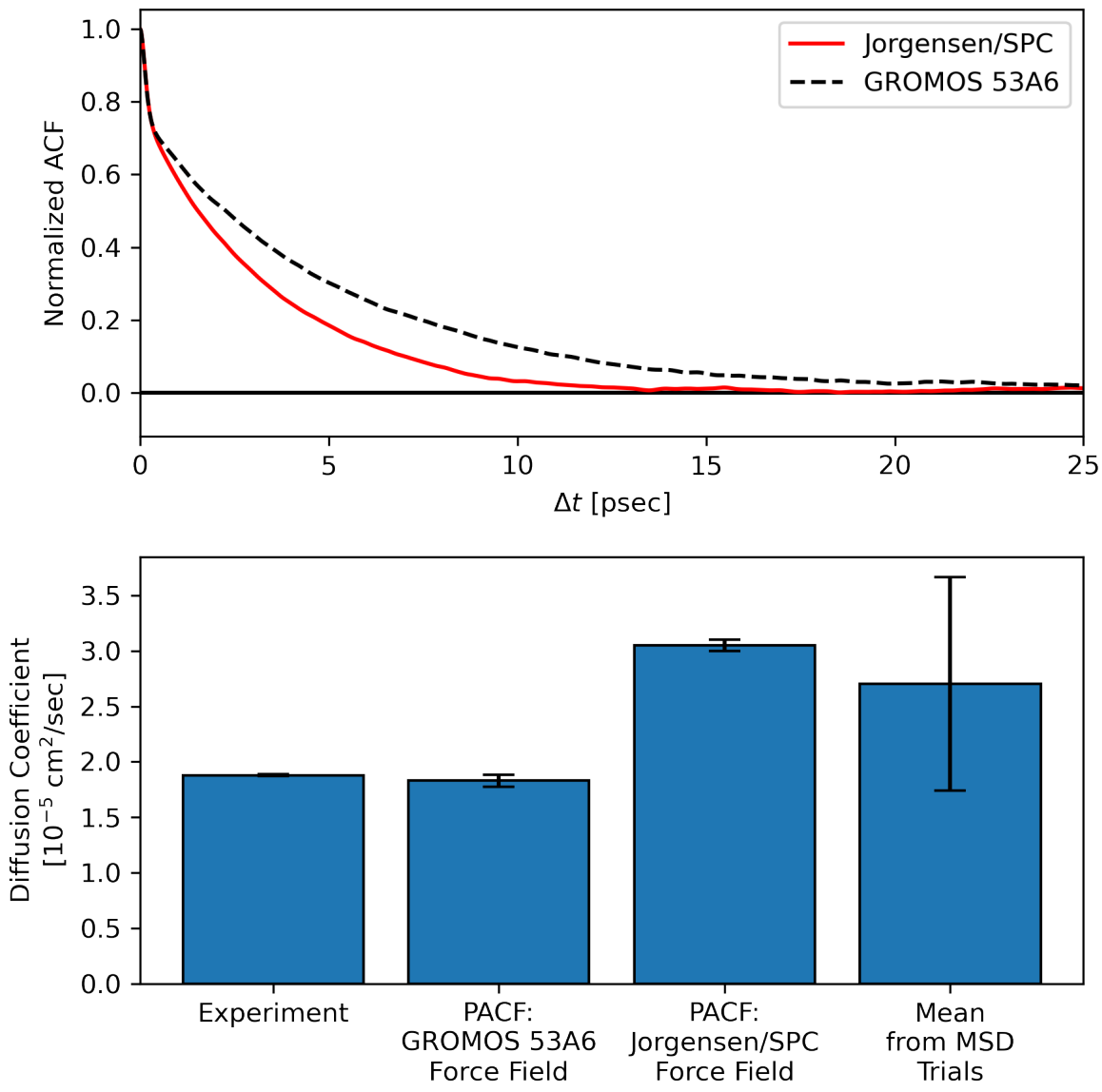

Figure 2: Upper panel: Comparison of Position Autocorrelation Functions (PACFs) for methane in bulk water using two different force fields. The PACF values are normalized to their respective coordinate Mean Squared Displacement (MSD) values. Both curves are for the z-direction. Lower panel: Comparison of diffusion coefficient for methane in bulk water from the experimental results of Witherspoon and Saraf [16], the PACF method using two different force fields, and the MSD method. The error bars for the experimental result are the reported standard deviation from repeated experimental trials. Each restrained molecular dynamics (MD) simulation resulted in a measurement three orthogonal directions, with the arithmetic mean shown here. The error bars for the restrained MD simulation results are the standard error of the mean from the three orthogonal measurements. Three trials of the unrestrained MD simulation were conducted, with the arithmetic mean shown here and error bars representing the standard error of the mean from the three trials. 


\subsection{Effects of confinement in molecular dynamics simula- tions}

We conducted MD simulations of aqueous methane inside the silicate channel to investigate the influence of confinement on the transport behavior of aqueous methane. Here, we present the results for a fully protonated channel of $1.6 \mathrm{~nm}$ in width. Results for variations in the channel width and silicate surface protonation level are presented in Sections 3.5 and 3.6, respectively. Our expectation for the channel is that interactions between the water and the channel surfaces will result in a water structure differing from bulk conditions, which in turn will produce non-bulk transport behavior for the methane. To provide insight into the confined water structure, we first examine the water density within the channel. Water density calculations were performed using water trajectories from simulations containing only the silicate and water, in the absence of the methane solute. The water density within the simulation volume is shown in Fig. 3 for a single plane. A region of the channel containing areas of both high and low water density was selected for further MD simulations to map the spatial variations of both the local diffusion coefficient and the PMF.

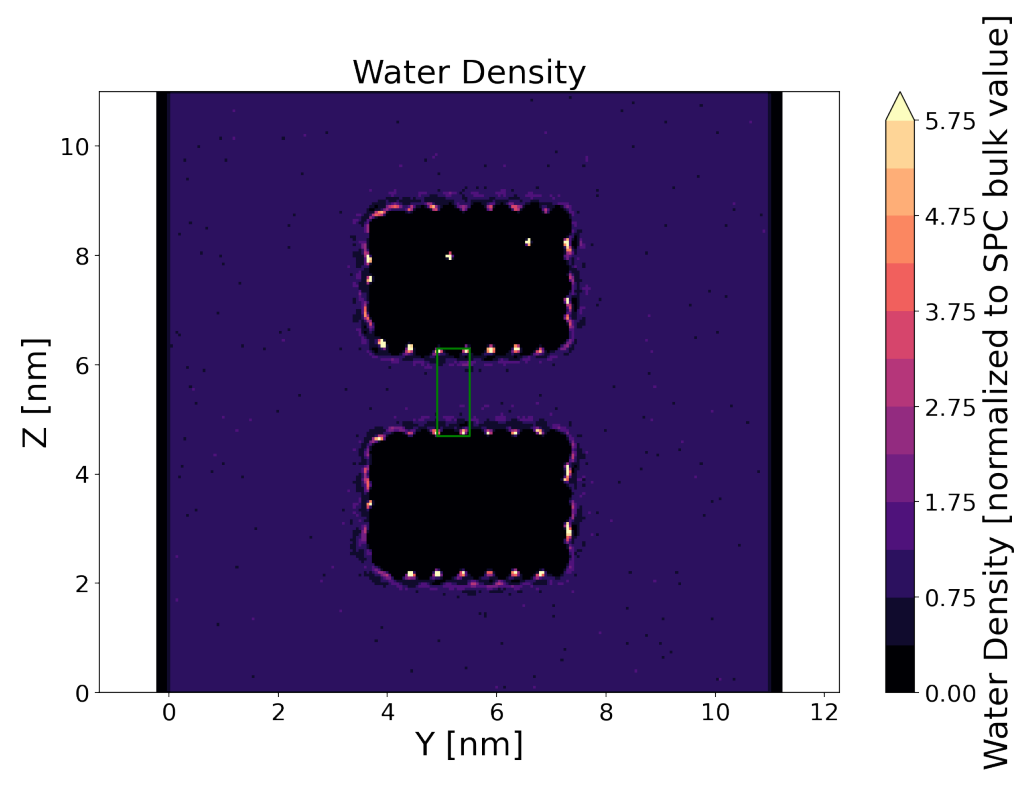

Figure 3: Water density over one plane in the MD simulation for the system shown in Fig. 3, with a $1.6 \mathrm{~nm}$ channel width between the two silicates. The dark areas of near-zero water density are the region occupied by the silicates. Large variations in the water density are observed near the silicate surfaces. The remainder of the model has a water density typical of bulk conditions. The green rectangle highlights the portion of the model selected for detailed measurement of the local diffusion coefficient and PMF. The water density was calculated at half- $\AA$ spatial resolution, from 128 ns of simulation data. 
Local diffusion coefficients were calculated within this region of interest as described in Sections 5.2 and 6.1.2. Fig. 4 shows the PACF results for three different locations within the channel. The curve for the bulk simulation presented in Section 3.3 (see upper panel of Figure 2) is also included for comparison. The curve that most nearly matches the bulk simulation result is from a point near the middle of the channel. Fig. 4 also shows the local diffusion coefficient results plotted against position within the channel. The local diffusion coefficient appears to approach the bulk value near the middle of the channel, and drop to roughly half this value at positions closer to the channel surfaces. This observation is consistent with the presence of solvation layers of water near the silicate surface, but a more bulk-like water structure near the middle of the channel.
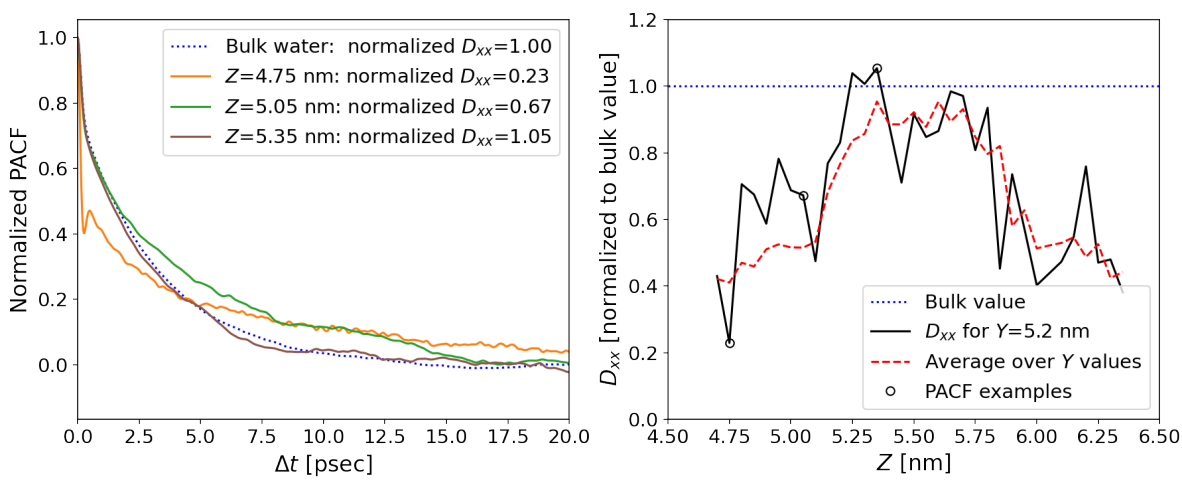

Figure 4: Examples of local diffusion coefficient in the $1.6 \mathrm{~nm}$ channel. Left: Position Autocorrelation Function (PACF) of methane for bulk conditions and three different positions in the channel with $Y=5.2 \mathrm{~nm}$. The PACF curve for the bulk condition is from a 16 ns simulation, while the channel simulations were 2 ns long. This results in a smoother curve for the bulk condition. Right: Local diffusion coefficient variation within the channel, showing the values along a line across the channel ( $z$-direction), and also the averages over lines parallel to the channel face ( $y$-direction). The locations that have PACF curves on the left panel are marked by circles on the right panel. The coordinate system is as shown in Figure 1. 
Fig. 5 illustrates the spatial variation of the PMF, local diffusion coefficient, and water density within the $1.6 \mathrm{~nm}$ channel. The values are averaged in the $y$-direction, to demonstrate the general trend in values across the channel. The local diffusion coefficient is suppressed near the channel walls, and approaches, but does not match, the bulk diffusion behavior near the center of the channel. This variation in the local diffusion coefficient is symmetric about the channel. In contrast, the PMF appears to be asymmetric within the channel, though still suppressed near the surfaces. This asymmetry may be the result of the asymmetry of the silicate faces themselves; the faces are not mirror images of one another. Also, the nearest silanol groups at each face are centered at different distances from the plane of consideration. Some of the asymmetry in the PMF may also be due to noise that could be reduced by longer simulations at each point of measurement. The water density seems to oscillate near the channel walls, likely due to solvation layers around the silicate, but the strength of this oscillation reduces quickly with distance from the surface. These patterns suggest that near the silicate surface, alterations of the water structure coincide with the alterations of the local diffusion coefficient and PMF.

A more detailed examination of the co-variation in the water density, local diffusion coefficient, and potential of mean force is presented in the contour plots of Fig. 6, which illustrate the variations of all three quantities over two spatial dimensions of the channel. The water density data is the same as shown in Fig. 3, but over a smaller region of the plane, centered near the area where measurements of the PMF and local diffusion coefficient were obtained. The water density is near its bulk value over most of the channel, but has some localized areas of high water density at nearly consistent spacing along the channel surfaces. This is most likely due to the presence of hydrophilic silanol groups present on the surface in repeating patterns. (The patterns of water density and the silicate face structure are compared in Figure S2.) The local diffusion coefficient results show the same pattern as the results in Figure 4 and 5: the value is highest near the middle of the channel, where the value is roughly the bulk diffusion coefficient, and then suppressed closer to the channel surfaces. The local diffusion coefficient seems to vary more with distance from the channel wall than with position along the channel. The PMF results are generally highest at the channel walls because the walls are impervious to methane. The PMF results are lower in the middle of the channel, where more bulk-like behavior is expected. However, localized depressions in the PMF seem to appear at the channel surface in a generally periodic arrangement. These depressions may be due to the periodic, but asymmetric, pattern of silanol groups on the silicate channel faces.

There is variation in the PMF even near the center of the channel, where the water density and local diffusion coefficient are most bulk-like. The variation of the PMF in the central region of the channel is roughly $1 k_{B} T$ in magnitude. For comparison, Bui et al. [8] obtained PMFs from Weighted Histogram Analysis Method (WHAM) for gas-phase methane diffusing through a hydrated silica channel of $1 \mathrm{~nm}$ width. In their results, the PMF at the center of the channel oscillated with an amplitude of roughly $0.8 k_{B} T$, which is comparable to our observations for aqueous methane near the center of a wider channel. 

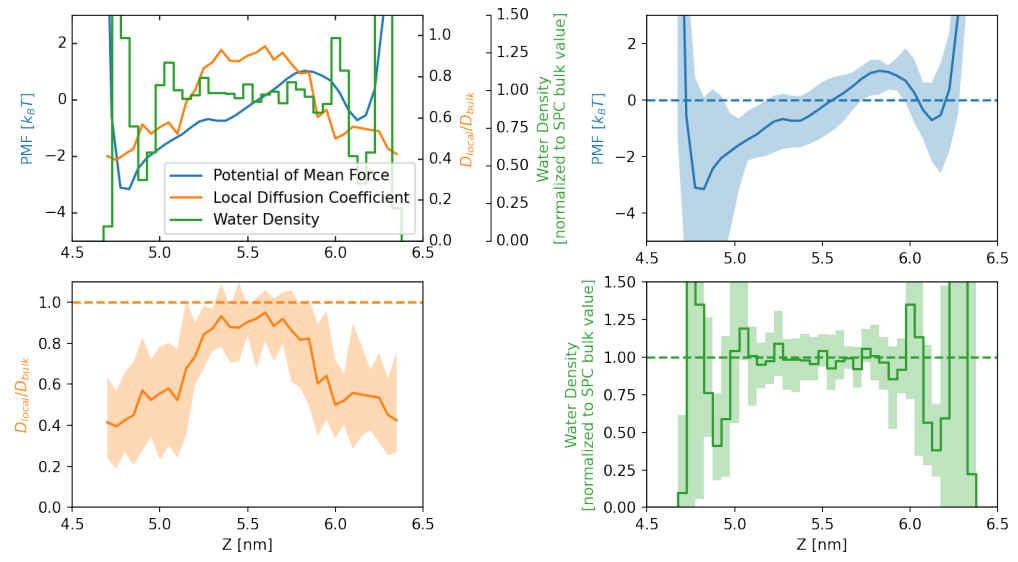

Figure 5: Spatial variation of averaged PMF, local diffusion coefficient, and water density in the $1.6 \mathrm{~nm}$ channel. The values are averaged over $0.6 \mathrm{~nm}$ in the $y$-direction, for a single value of $x$, showing the spatial variation in the $z$ direction (perpendicular to the channel faces). Dashed lines show the values under bulk conditions. Shaded bands show the limits of variation in the $y$ direction. 

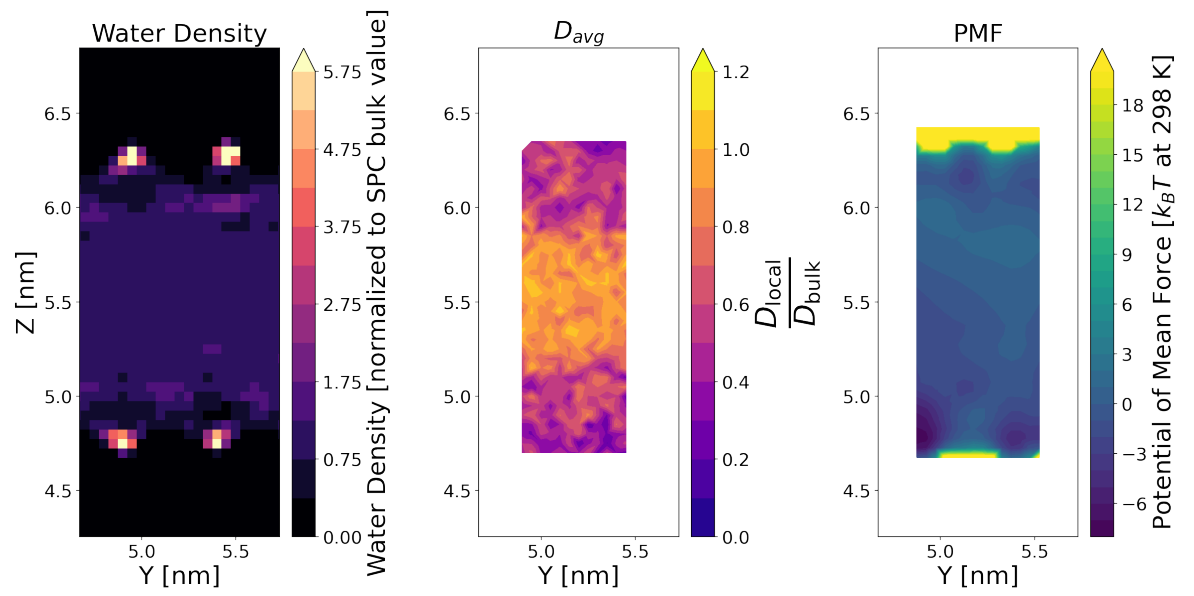

Figure 6: Water density, local diffusion coefficient, and potential of mean force for the $1.6 \mathrm{~nm}$ channel width, fully protonated. The imposed rectangle shows the bounds of the data selected for the Finite Element model within the plane. The water density calculation is from $128 \mathrm{~ns}$ of simulation data, at half- $\AA$ spatial resolution. 


\subsection{Effects of variation in silicate porosity}

To assess the influence of porosity on transport behavior within the silicate channel, MD simulations were conducted for channel widths of $1.2,1.6$, and 2.0 $\mathrm{nm}$. Results for water density, PMF, and local diffusion coefficient for these channel widths are shown in Figure 7 and in Figure S3 in the Supplement. The water density follows a similar pattern for all three channel widths: the density is very nearly the value under bulk conditions over most of the channel width, but increases at select locations near the silicate surface. The water density appears to oscillate with distance from the silicate face, with the amplitude of the oscillations decreasing with distance. Such oscillations of the water density are somewhat indicative of solvation shells around the silicate. The PMF results generally follow a pattern of approximating the bulk value near the middle of the channel, though localized areas of low PMF adjacent to the silicate surface are sometimes present. The local diffusion coefficients also follow a similar pattern in all three channel widths: the coefficient is highest near the middle of the channel, and drops to a lower value at locations closer to the silicate faces. However, the local diffusion coefficients near the middle of the channel are closer to the bulk value for the larger channel widths. These results illustrate that the suppression of the local diffusion coefficient near the channel walls is consistent for different channel sizes. Suppression of the effective diffusion coefficient is expected, though it seems the size of the effect should generally reduce with increasing channel width. 

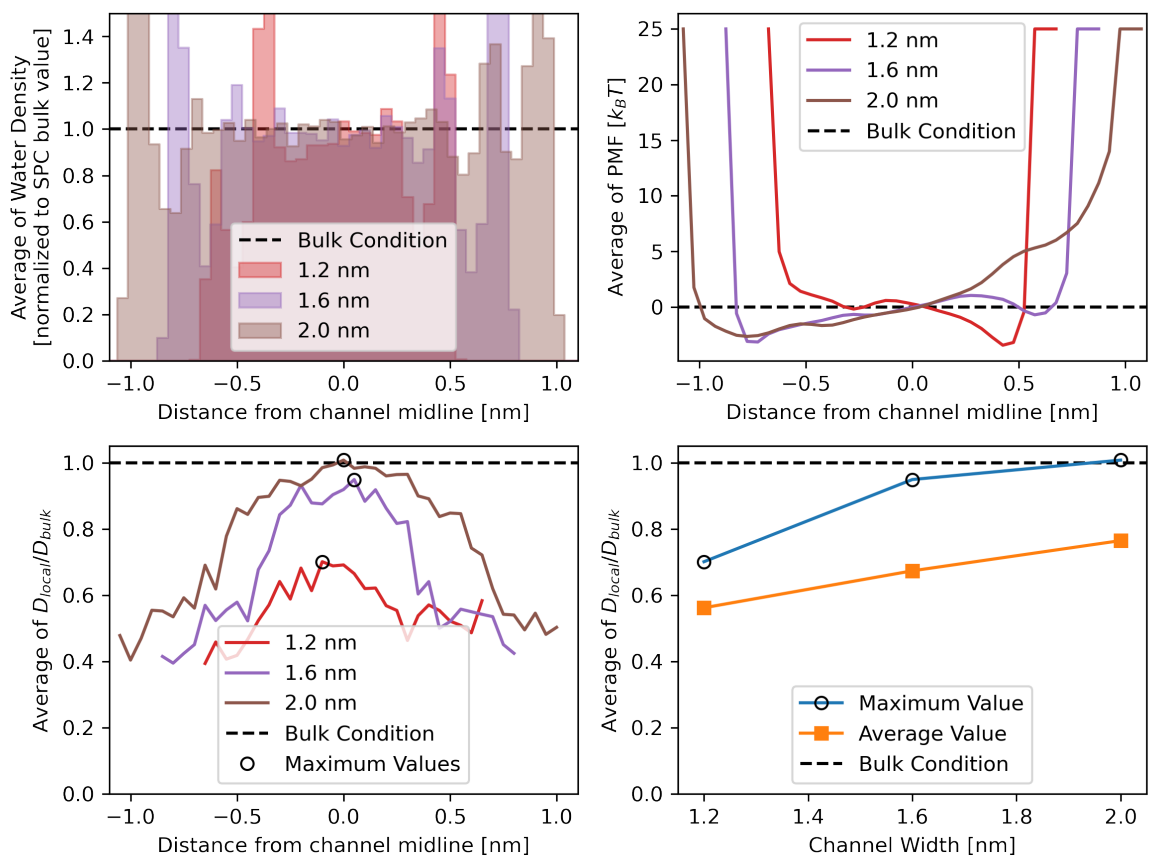

Figure 7: Water density, PMF, and local diffusion coefficient results for channel widths of $1.2,1.6$, and $2.0 \mathrm{~nm}$. These results are averaged over the $y$ direction to show variation across the channel. All silanols are fully protonated for these simulations. Top left: water density. Top right: PMF, with zero as the bulk potential value. Lower left: local diffusion coefficient, normalized to the bulk value. Lower right: maximum values of the normalized local diffusion coefficients for each channel width, and the spatial average of the normalized local diffusion coefficients. 


\subsection{Effects of variation in surface protonation levels}

To assess the influence of the silicate surface chemistry on transport behavior within the channel, MD simulations were conducted for surface a range of surface protonation levels. Specifically, simulations were conducted with $100 \%, 73 \%$, and $50 \%$ of the silanol groups on the silicate surface protonated, as structures for these protonation levels were provided in [14, 15]. In the de-protonated silanol groups, the hydrogen is absent, leaving a negative charge on the oxygen ion. To balance this charge, one sodium ion was added to the simulation for each de-protonated silanol group. These simulations used a channel width of $2.0 \mathrm{~nm}$. Results for water density, PMF, and local diffusion coefficient for these channel widths are shown in Figure 8 and in Figure S3 in the Supplement. The $\mathrm{PMF}$ and local diffusion coefficient values generally seem to be consistent with a slight reduction in the effective channel width as the level of protonation is decreased. A similar pattern, though less pronounced, can also be inferred in the water density results. This effect may be produced by sodium ions residing at some of the deprotonated silanol locations, potentially creating an obstacle to methane diffusion as well as further changes to the local water structure. It is possible that this effect would be more pronounced at smaller channel widths than $2.0 \mathrm{~nm}$. Furthermore, while the PMF for the fully protonated condition has a region of negative value, representing an area attractive to methane, this region is absent in both of the partially de-protonated cases. This change in the PMF will later be seen to have a great deal of impact on the effective diffusion coefficients presented in Sect. 3.7. 

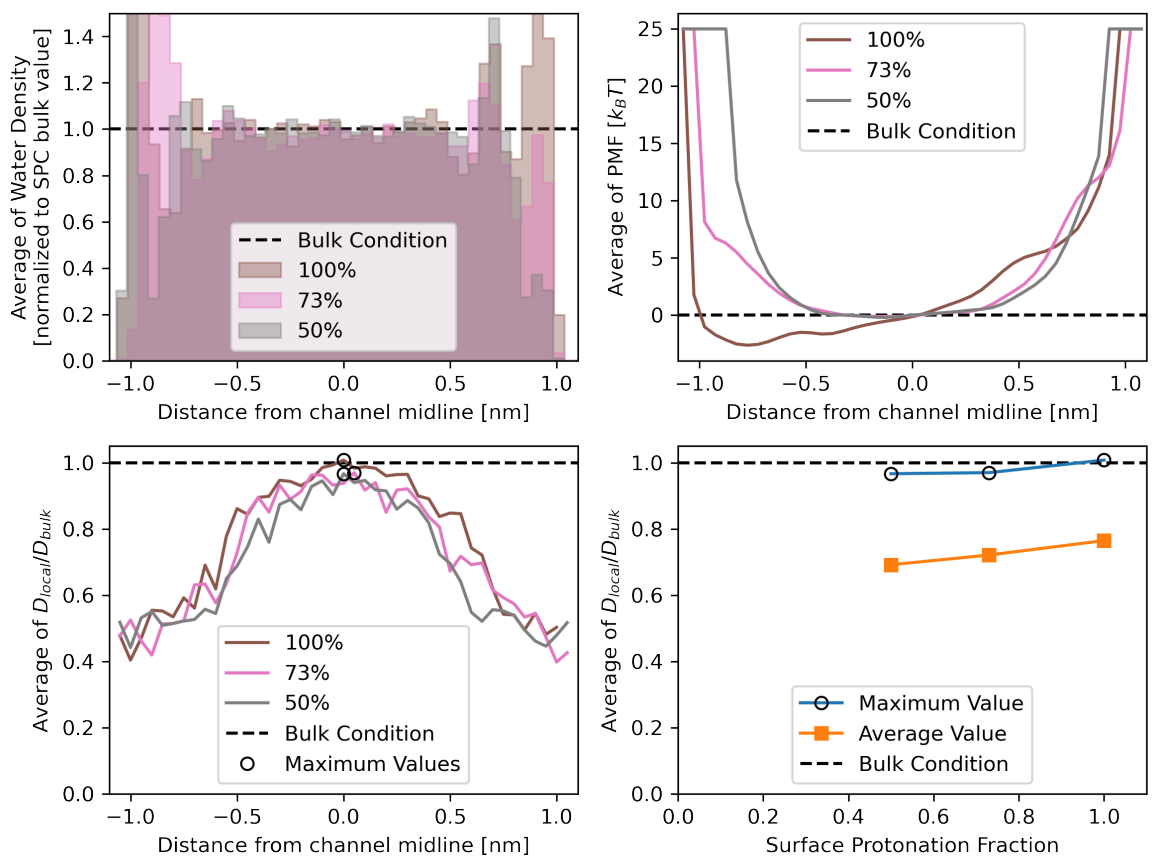

Figure 8: Water density, PMF, and local diffusion coefficient results for surface protonation levels of $100 \%, 73 \%$, and $50 \%$. These results are averaged over the $y$ direction to show variation across the channel. All channels shown are $2.0 \mathrm{~nm}$ in width. Top left: water density. Top right: PMF, with zero as the bulk potential value. Lower left: local diffusion coefficient, normalized to the bulk value. Lower right: maximum values of the normalized local diffusion coefficients for each deprotonation level, and the spatial average of the normalized local diffusion coefficients. 


\subsection{Homogenization based on non-bulk behavior in the channel}

We next incorporated the spatially varying local diffusion coefficients and PMF values from the MD simulations into the homogenization simulations, in order to assess the impact of the observed differences from bulk behavior into the predicted transport properties of the nanoporous silicate. Variations in both the channel width and the surface protonation level, identical to those in the MD simulations, were included in the homogenization calculations. The results of these simulations are shown in Figure 9. The theoretical calculations described in Sections 3.1 and 3.2 are shown in the figure, along with the results of homogenization calculations for the same scenarios. As indicated previously, the homogenization simulations reproduced the expected results for these situations.

The results from the left panel of Figure 9 generally show an increase in the effective diffusion coefficient with increasing channel width in all scenarios, due to the corresponding increase in porosity. Compared to the scenario where the diffusion coefficient inside the channel is assumed to match the bulk value in all locations (dashed red line), the use of the local diffusion coefficient from MD (solid red line) reduces the effective diffusion coefficient by approximately $23-43 \%$, with the reduction being larger for smaller channel widths. This is presumably due to the suppression of the local diffusion coefficient near the channel walls noted previously. In contrast, maintaining the bulk diffusion coefficient in the channel but adding in a PMF with regions that are more attractive to methane than the bulk condition can increase the effective diffusion coefficient. For the PMF from MD simulations (dashed blue line), this increase was approximately $200-283 \%$. For the quadratic PMF with the same maximum and minimum values (dashed green line), this increase was approximately $40 \%$ for each channel width, illustrating the importance of the spatial variations in the PMF rather than only its magnitude alone. These competing effects of a decrease due to the local diffusion coefficient, and an increase due to the PMF combined (solid blue line) result in an overall increase of the effective diffusion coefficient of about $84-112 \%$. It appears that the PMF more strongly influences the effective diffusion coefficient than does the local diffusion coefficient. In the right panel of Figure 9, the assumption of bulk conditions within the channel (dashed red line) prevents any effects of the surface protonation level on the effective diffusion coefficient, resulting in a horizontal line. Using the local diffusion coefficient values from MD without a PMF (solid red line) results in an overall reduction of the effective diffusion coefficient, again due to the suppression of the local diffusion coefficient near the channel walls. Interestingly, the reduction is slightly more pronounced for lower levels of surface protonation, with about a $10 \%$ reduction from the fully protonated case to the $50 \%$ protonated case. This suggests that the local diffusion coefficient is slightly more suppressed at lower solvent $\mathrm{pH}$ levels, which is consistent with the data shown in Figure 8. Most significantly, while the use of the PMF from MD (blue lines) results in an increase in the effective diffusion coefficient for the fully protonated case compared to the cases without a PMF (red lines), the partially de-protonated cases experience a reduction in the effective diffusion coefficient instead. The reduction for the partially de-protonated cases with a PMF (blue lines) is about $40-60 \%$ for the corresponding cases without a PMF (red lines). For the cases with a PMF (blue lines), there is a reduction of about $85 \%$ from the fully protonated case to the partially de-protonated cases. Thus, the the surface protonation level appears to influence the effective diffusion coefficient more strongly through its effects on the PMF within the channel than through changes to the local diffusion coefficient. 

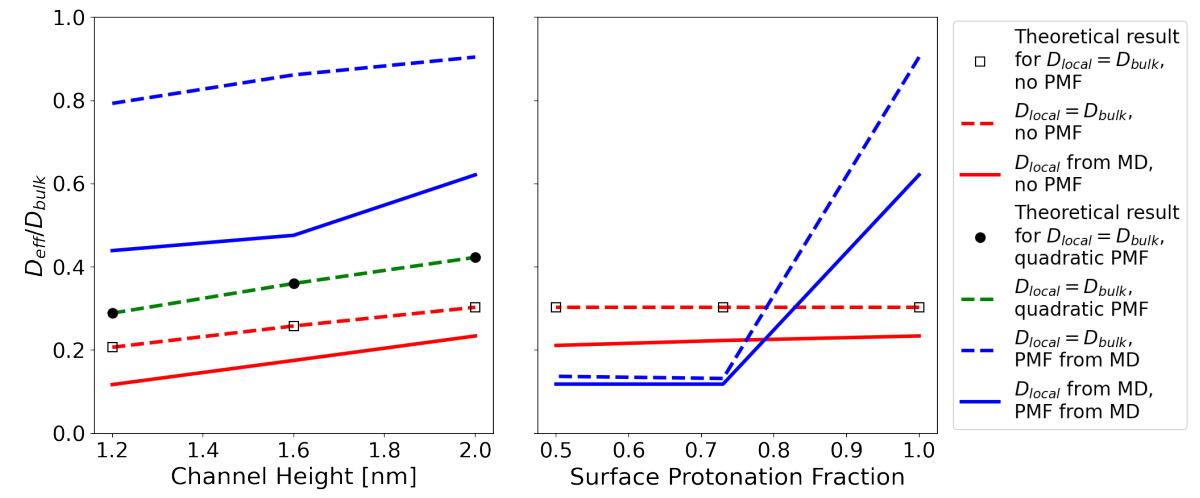

Figure 9: Effective diffusion coefficient predictions from homogenization simulations of the silicate channel. The results are plotted as an effective diffusion coefficient normalized to the bulk diffusion coefficient. Left panel: Results for channels of varying porosity, with all surfaces fully protonated. Right panel: Results for channels of $2.0 \mathrm{~nm}$ width, with different levels of surface protonation. Results from homogenization simulations are shown with continuous lines, while analytically-derived results are plotted as points. Dashed lines are for simulations where the local diffusion coefficient is spatially invariant and equal to the bulk diffusion coefficient. Solid lines are for simulations where the local diffusion coefficient was taken from the MD results. Red lines are for simulations without a PMF. The green line is for simulations with a quadratic potential as described in Sect. 3.2. Blue lines are for simulations where the PMF was taken from the MD results. 


\section{Discussion and conclusion}

\subsection{Discussion of results}

Our hypothetical explanation for the observed results is that the silicate surface induces solvation layers in the water within the channel. This water structure, in turn, influences local diffusion coefficients with the channel and also creates regions of space that are more favorable and less favorable to the presence of methane, which can be expressed as a PMF. These spatial variations in the transport properties within the channel then contribute to changes in the effective diffusion coefficient for the silicate material as a whole. In this way, the interactions at the atomistic scale can be incorporated into multi-scale theory for the diffusion coefficient appropriate at analysis scales representing hundreds or thousands of nanometers.

The oscillations of decreasing amplitude in the water density seem to be consistent with the idea of solvation layers around the silicate, and previous observations of water layers in confined systems [10]. The decrease of the amplitude with distance from the silicate surface suggests that the closer solvation layers are more strongly defined than more distant ones, in a transition to water bulk behavior. This layered structure of the water may also be responsible for reduced methane transport near the channel surfaces. Our initial expectation was that methane methane would be attracted to areas of low water density and repelled from areas of high water density because methane by the hydrophobic interaction. However, the observed data does not generally match this expectation. Indeed, the layered water structure is not apparent in the PMF data.

The results of the homogenization simulations are consistent with other theoretical predictions for simple situations where such predictions were possible. The local diffusion coefficients in the MD results were found to be lower near the silicate surfaces, resulting in a reduction of the effective diffusion coefficient that varied with channel width and surface protonation level. The changes in the effective diffusion coefficient created by using the local diffusion coefficient and PMF from MD results were also found to be appropriate for the MD results with varying channel width and surface protonation level. The homogenization simulations suggest that both the local diffusion coefficient and the PMF inside the channel significantly influence the effective diffusion coefficient, and are both needed for accurate theoretical predictions.

Overall, the MD and homogenization results illustrate the assumption of bulk behavior within the channel is not justified and can lead to significant errors, even though the non-bulk behavior within the channel includes competing factors that partially offset one another. Furthermore, the results are consistent with findings of Collin et al. [10] that the surface chemistry of the channel can have a significant influence on the transport behavior. The influence of the surface chemistry can be seen not only at the atomistic scale, but also at larger analysis scales through the effective diffusion coefficient of the silicate material.

\subsection{Assumptions and limitations}

A number of assumptions were necessary to complete these studies. A harmonic restraining potential was applied to all atoms of the silicate in order to remove rigid-body motion of the silicate that would alter the channel geometry. This includes the surface atoms of the silicate, potentially altering their response to the presence of water and methane. We assume that any such effects on the interactions between the silicate and the water or methane would be small in comparison to the solvation effects under study.

The WHAM approach for PMF measurement within the channel provides a spatially smooth result. In contrast, the water density and local diffusion 
coefficient measurements within the channel include some amount of noise, as would be expected in data from MD simulations. Such noise is presumably also present in the PMF, despite the apparent smoothness of the data. Our interpretation of the PMF data assumes that this noise is small in comparison to the real spatial variations, just as is the case for the water density and local diffusion coefficient values.

As noted in Sect. 5.2, the constant $K$ representing the strength of the restraining potential for the methane must simultaneously meet a few different requirements. The value must be small enough to maintain the assumption of over-damped dynamics. The values appropriate for this assumption may depend on the non-bonded interaction parameters for the methane and water in the MD force field. The value must also be large enough to adequately confine the molecule for appropriate spatial resolution of the local diffusion coefficient, as the region of space sampled by the methane molecule decreases with increasing $K$. Finally, the value must also be larger than the magnitude of the corresponding local PMF gradient by an amount sufficient to justify neglecting the PMF in the post-processing of the PACF. Further challenges in the selection of $K$ have previously been noted by other researchers [9, 19]. The results herein assume that there is a range of values of $K$ where all of these requirements are simultaneously met, and that the selected value is within this range. In particular, this assumption is most challenged for methane locations near the silicate channel surface, where the PMF gradient has a large magnitude.

As noted in Sect. 3.3, the force field parameters used in the MD simulations were not able to fully match the established empirical value of the diffusion coefficient for methane in bulk water. Our results assume that the difference between the empirical value and the value measured in the MD simulations in linearly proportional to the simulation result, such that normalization by the simulation bulk value is sufficient to generally resolve the discrepancy.

\subsection{Conclusion}

The transport behavior of aqueous methane through porous catalysts may play a crucial role in the future design of gas-to-liquid methane conversion processes. Describing this behavior requires assessments of atomistic-level and continuumlevel phenomena that jointly influence substrate transport. With respect to atomistic considerations, this work has demonstrated that the forces involved in diffusion can be resolved using MD simulations, by measuring the diffusion coefficient and the PMF. The techniques employed herein can be applied even when both of these parameters have spatial variations. Importantly, we show that homogenization simulations are well-suited to couple atomistic-scale behavior to the continuum level, namely by extrapolating these spatial variables to obtain effective transport properties for a continuum approximation of a porous medium. As noted in the results above, changes in the material porosity and the surface chemistry of the pores can have effects on the local diffusion coefficient and PMF within the pore. These changes ultimately impact the effective diffusion coefficient as well, allowing for the tuning of transport properties by the proper selection of material porosity and surface chemistry. Further, these techniques could also be extended to biological systems that are strongly influenced by molecular-level interactions, as examined in some of our previous work $[21,22]$.

\section{Theory}




\subsection{Hypothesis for the influence of solvent density on local transport behavior}

The motion of a single methane molecule surrounded by a solvent such as water can be described by a Langevin equation in the limit of strong friction:

$$
\xi \dot{x}=-\frac{\partial}{\partial x} V(x)+f(t)
$$

where $\xi$ is the friction coefficient representing solute/solvent interactions, $V(x)$ is a mean force potential, and $f(t)$ is a force varying randomly in time. A single independent variable is shown here for clarity, but the analysis generalizes easily to higher dimensions, as there is no coupling between orthogonal components of the motion. When the solvent is confined to nano-scale pores within a medium such as a silicate, the surface chemistry of the pores could cause local variations in the number density of the solvent molecules. Our expectation is that these local variations in the solvent density will alter the local transport properties of the methane. We describe these effects by making the friction and potential terms of the Langevin equation spatial variables.

Through the Einstein relation between $\xi$ and the diffusion coefficient, $D$, spatial variations in the friction coefficient will produce spatial variations in $D$ as well. This spatial variation was measured in the MD simulations using the approach described in Section 5.2. The spatially varying PMF was measured using standard procedures described in Section 6.1.2.

\subsection{Determination of local diffusion of coefficient from MD simulation}

The diffusion coefficient relates the flux of a diffusion species to its concentration gradient. In homogeneous media the average diffusion can be estimated based on the MSD of individual molecules:

$$
D=\frac{1}{6} \frac{\partial\left\langle r^{2}\right\rangle}{\partial t}
$$

This approach is not applicable to non-homogeneous media, as the MSD would be influenced by the local diffusion coefficient in all the areas visited by the diffusing molecule. Instead, the spatial variation of the transport coefficient can be obtained from a Green-Kubo relation. In our model, MD simulations (described in Sect. 6.1) were used to predict Eq. 16 parameters using an approach described in Daldrop, Kowalik, and Netz [19], based on earlier work in Hummer [23] and Woolf and Roux [24]. We also previously used this approach in Setny et al. [25]. The self-diffusion coefficient for the solute methane here implicitly reflects the solute/solvent (water) interactions. Namely, the motion of methane is approximated as over-damped Brownian motion.

To obtain local values of the diffusion coefficient, the methane is restrained by a harmonic potential $\frac{1}{2} K x^{2}$. The equation of motion for the methane molecule is therefore the Langevin equation of Eq. 5.

$$
\xi \dot{x}=-K x+f(t)
$$

The statistical properties of the random variation of $f(t)$ are such that they satisfy Eq. 6 [26-28].

$$
\begin{aligned}
\langle f(t)\rangle & =0 \\
\langle f(0) f(t)\rangle & =2 \xi k_{B} T
\end{aligned}
$$


Under these conditions, it can be shown that the solution for $x(t)$ will have the properties shown in Eq. 7.

$$
\begin{aligned}
\left\langle x^{2}\right\rangle & =\frac{k_{B} T}{K} \\
\langle x(0) x(t)\rangle & =\left\langle x^{2}\right\rangle e^{-t / \tau}
\end{aligned}
$$

where $\tau=\xi / K$. From these results, the integral of the position autocorrelation function is given by Eq. 8 .

$$
\tau=\frac{1}{\left\langle x^{2}\right\rangle} \int_{0}^{\infty}\langle x(0) x(t)\rangle d t
$$

This allows for determination of the friction coefficient $\xi$, and thus also the local diffusion coefficient $D$, using the Einstein relation of Eq. 9.

$$
D=\frac{k_{B} T}{\xi}=\frac{\left\langle x^{2}\right\rangle}{\tau}
$$

In Eq. 9, the actual MSD value, $\left\langle x^{2}\right\rangle$, is used in place of its theoretical value for a harmonic potential, because the actual potential is the superposition of the harmonic biasing potential and the PMF. The procedure is adopted here, under the assumption that the gradient of the PMF will be small in comparison to the value of $K$ for the restraining potential. Note that larger values of $K$ will confine the methane to a smaller region around the center of the harmonic potential, allowing spatial variations in the local diffusion coefficient to be measured with greater spatial precision. However, large values of $K$ can also invalidate the assumption of over-damping. This can result in oscillatory behavior in the PACF, making integration more difficult [19]. Here, the value of $K$ for the harmonic potential was selected with the aim of being small enough to maintain over-damped behavior, while remaining larger than reasonable magnitudes for the PMF gradient.

The center of the harmonic restraining potential can be shifted to obtain the local diffusion coefficient at selected spatial locations.

For anisotropic conditions, this analysis can be repeated for each component of the position autocorrelation function to obtain the diagonal matrix components. The simple geometry of the silicate channel studied here makes it very likely that the selected coordinate system is a principal coordinate system, where the off-diagonal terms of the matrix are zero. Future applications of this method may involve porous materials with more complex pore geometry, where the offdiagonal terms may be nonzero in some locations. A relatively minor extension of the method for such circumstances would be to rotate the coordinate system of the methane trajectories prior to calculation of the PACF, allowing the diagonal matrix terms to be computed in a different coordinate system. An estimate of the off-diagonal terms could then be obtained from the known transformation matrix between these two coordinate systems.

\section{Methods}

\subsection{Molecular dynamics}

\subsubsection{Parameterization and simulation}

Molecular dynamics simulations were conducted in GROMACS. The OPLS-AA SPC water model was used for the explicit solvent. The united atom approximation was selected for the methane molecule, on the basis of the results presented in Bhatia and Nicholson [29]. The nonbonded force field parameters for 


\begin{tabular}{ccccc}
\hline Species & $\sigma[\mathrm{nm}]$ & $\epsilon[\mathrm{kJ} / \mathrm{mole}]$ & $q[\mathrm{e}]$ & Reference \\
\hline $\mathrm{Si}$ & 0.37 & 0.39 & +1.1 & Emami et al. [30] \\
$\mathrm{O}_{\mathrm{Si}}$ & 0.31 & 0.23 & -0.55 & Emami et al. [30] \\
$\mathrm{O}_{\mathrm{H}}$ & 0.31 & 0.51 & -0.68 & Emami et al. [30] \\
$\mathrm{H}$ & 0.096 & 0.063 & +0.4 & Emami et al. [30] \\
$\mathrm{CH}_{4}$ & 0.37 & 1.23 & 0.0 & Jorgensen, Madura, and Swenson [31] \\
\hline
\end{tabular}

Table 5: Non-bonded interaction parameters used for molecular dynamics simulations in GROMACS.

methane were taken from Jorgensen, Madura, and Swenson [18], which used the same functional form for the 12-6 Lennard-Jones potential as GROMACS, so that only unit conversions of the parameters were required. While the combining rule noted in Jorgensen, Madura, and Swenson [18] is the geometric average, the arithmetic average is used herein. The force field parameters for silica were taken from Emami et al. [14, 15], and converted to the units and functional form used in GROMACS. Specifically, the functional form of the 12-6 Lennard-Jones potential for nonbonded interactions in Emami et al. [14] is given by Equation 10, while the functional form used by GROMACS is shown in Equation 11. In both equations, $E$ is the interaction energy, and $r$ is the separation distance between the two atoms. The two functional forms can be used to represent the same interaction potential by computing the GROMACS parameters from the parameters provided by Emami et al. [14] using the relationships of Equation 12.

$$
\begin{gathered}
E=\epsilon_{\text {amber }}\left(\left(\frac{\sigma_{\text {amber }}}{r}\right)^{12}-2\left(\frac{\sigma_{\text {amber }}}{r}\right)^{6}\right) \\
E=4 \epsilon_{\text {gromacs }}\left(\left(\frac{\sigma_{\text {gromacs }}}{r}\right)^{12}-\left(\frac{\sigma_{\text {gromacs }}}{r}\right)^{6}\right) \\
\epsilon_{\text {gromacs }}=\epsilon_{\text {amber }} \\
\sigma_{\text {gromacs }}=2^{-1 / 6} \sigma_{\text {amber }}
\end{gathered}
$$

The nonbonded interaction parameters used in the simulations are listed in Table 5 .

To confirm the proper conversion of the nonbonded force field parameters, the same method was used to convert the parameters from an AMBER input file to the corresponding GROMACS input file parameters for an AMBER force field. This comparison involves an additional step, because the AMBER force field parameters specify the van der Waals radius of each atom, and the depth of the potential well, rather than specifying $\sigma_{\text {amber }}$ and $\epsilon_{\text {amber }}$ directly. The additional calculation is relatively simple, because the functional form of Equation 10 used by AMBER has a well depth that is equal to $\epsilon_{\text {amber }}$, with the point of minimum potential energy located at $r=\sigma_{\text {amber }}$. At this minimum, the separation distance $r$ between two identical atoms would be twice the van der Waals radius. Thus, $\sigma_{\text {amber }}$ is simply twice the specified van der Waals radius, and $\epsilon_{\text {amber }}$ is equal to the depth of the potential well. Using this information, the gromacs parameters for the AMBER96 and AMBER99 force fields were successfully reproduced.

Following solvation, energy minimization was performed for 50,000 steps, and then velocities were randomly assigned according to a Maxwell distribution. The system was equilibrated in the Number, Volume, Temperature thermodynamic ensemble (NVT) ensemble for 0.1 nanoseconds, using the Nosé-Hoover thermostat. This was followed by 0.1 nanoseconds of equilibration in the Num- 
ber, Pressure, Temperature thermodynamic ensemble (NPT) ensemble, using the Berendsen barostat and modified Berendsen thermostat. Production runs followed, using the NVT ensemble with the Nosé-Hoover thermostat. The production runs had a duration of 2 nanoseconds, except where noted otherwise. In all analysis steps, electrostatic interactions were evaluated using the Particle Mesh Ewald (PME) method [32, 33]. The dynamic runs used a time-step of 2 femtoseconds, with hydrogen bonds converted to constraints maintained by the LINCS algorithm [34].

The harmonic restraining potential applied to the methane molecule used a $K$ of $3.00 \times 10^{2} \mathrm{~kJ} \mathrm{~mol}^{-1} \mathrm{~nm}^{-2}$, for each direction. To prevent global translations, rotations, or deformations of the silicate bodies within the simulation unit cell, each atom of the silicate material was placed in a harmonic restraining potential with $K$ of $1.000 \times 10^{3} \mathrm{~kJ} \mathrm{~mol}^{-1} \mathrm{~nm}^{-2}$, for each direction. This includes the hydrogen atoms on the surficial silanol groups, which have affected the solvent-surface interactions.

\subsubsection{Processing methods}

Simulations of the solvated silicate channel without methane were used to calculate the spatial variation of water density within the channel. Water density was calculated from the water molecule trajectories using the MDAnalysispackage [35, 36] (which uses numpy [37]). The production runs for the water density calculations were 128 nanoseconds in duration.

Based on the water density results, portions of the channel were selected for detailed measurement of the local diffusion coefficient and PMF for methane. An $x$-plane was chosen, with dimensions in the $z$-direction fully extending across the channel from one silicate face to the other. The selected area also extended 0.6 nanometers in the $y$-direction. A different region was selected for each channel geometry and deprotonation level. The regions were chosen so as to include areas of both high and low water density at the channel boundaries.

PMF values were computed using the two-dimensional version of WHAM [38] (Version 2.0.10.1), which implements the WHAM [39-41]. The $y$ and $z$ coordinates of the methane or sodium molecule were used as the reaction coordinates in this approach.

The PMF values resulting from WHAM are relative values, with the minimum value in the data set originally chosen as zero. Instead, the analysis conducted here requires that a PMF value of zero represent the conditions of bulk water. Assuming that all studied channels are sufficiently wide that bulk behavior is recovered in the middle of the channel, this location can be used as a reference value for the PMF. After generation by WHAM, the values were shifted such that the average of the PMF values at the middle of the channel would be zero. Also, numerical difficulties were encountered in the homogenization simulations when using the PMF values directly from the MD simulations, even after adjusting to the gauge condition. To allow the simulations to complete successfully, the average value of the PMF was taken in the $y$-direction, such that the PMF used in the homogenization simulations varied only in $z$, which is the direction perpendicular to the silicate faces.

Local diffusion coefficients were calculated using the approach described in Sect. 5.2. PACFs were calculated by GROMACS, which returns the PACF function normalized by the MSD as illustrated in Equation 13.

$$
\text { normalized } \operatorname{PACF}(t)=\frac{1}{\left\langle x^{2}\right\rangle}\langle x(0) x(t)\rangle
$$

Observations of typical normalized PACF results showed that, in addition to the exponential decay predicted by Equation 7, there was also an short-duration Gaussian superimposed on the PACF for small values of time. Accordingly, the 
functional form in Equation 14 was selected as being a generally representative form, with fitting constants $A, \alpha$, and $\sigma$. The analytical integral of this form is shown in Equation 15.

$$
\begin{gathered}
\text { normalized } \mathrm{PACF}_{\mathrm{fit}}(t)=A e^{-t / \sigma}+(1-A) e^{-\alpha t^{2}} \\
\left.\tau=\int_{0}^{\infty} d t \text { (normalized } \mathrm{PACF}_{\mathrm{fit}}(t)\right)=A \sigma+\frac{1-A}{2} \sqrt{\frac{\pi}{\alpha}}
\end{gathered}
$$

Following calculation of a normalized PACF from methane trajectory data, a python script using the numpy package [37] fit the analytical function of Equation 14 to the normalized PACF data. The analytical integration of the normalized PACF was then calculated from Equation 15. To confirm a successful fit, the integral of the normalized PACF was also obtained numerically using the trapezoidal rule over the first 25 picoseconds. Cases where the numerical and analytical integrations differed by more than $25 \%$ were discarded from the data set.

The diffusion coefficients computed from the methane PACFs were generally consistent for the $x$ and $y$ directions, but different in the $z$ direction. Ultimately, the effective diffusion coefficient for only the $x$ and $y$ directions is desired, as the $z$ direction would be impervious. Furthermore, the channel itself is identical in the $x$ and $y$ directions, so the expected results for these two directions would be identical. Accordingly, the homogenization process used an isotropic local diffusion coefficient, which was computed as the arithmetic average of the $x$ and $y$ results from the MD simulations.

As noted in Section 3.3, the local diffusion coefficient results from MD were normalized to the bulk value predicted by the same method. In any cases where the normalized result was greater than 2.0, the result was limited to this value.

Homogenization theory is intended for application to periodic functions. Because the local diffusion coefficients and PMF values from the MD simulations were not periodic, they were reflected about both their right and upper boundaries to produce a symmetric unit cell for homogenization.

\subsection{Finite element modeling}

\subsubsection{Continuum level modeling of aqueous methane diffusion in sil- ica material}

Diffusion is represented in the continuum model by Fick's law [42] which can be written as

$$
\frac{\partial c}{\partial t}=\frac{\partial}{\partial x_{i}} D_{i j} \frac{\partial c}{\partial x_{j}}
$$

where

- $c$ represents the concentration of methane as a function of space and time

- $t$ represents time

- $x_{i}$ represents one of the three spatial coordinates

- the summation convention is used (a repeated index in any term implies summation over all three dimensions for that index)

- $D_{i j}$ represents the diffusion matrix, with three rows and three columns

When the diffusive medium is isotropic and spatially invariant, the diffusion matrix is simply the identity matrix scaled by a diffusion constant, $D$, and Fick's law can be written as 


$$
\frac{\partial c}{\partial t}=D \nabla^{2} c
$$

Eq. 17 can be generalized to reflect the influence of a time-independent and spatially-heterogeneous mean potential field, $V(x)$, using the Smoluchowski equation shown in Eq. 18. The Smoluchowski equation can be derived from the Langevin equation of Eq. 5 under appropriate assumptions [43, 44].

$$
\frac{\partial c}{\partial t}=\nabla \cdot\left(D e^{-\frac{V}{k_{B} T}} \nabla\left(c e^{\frac{V}{k_{B} T}}\right)\right)
$$

This can be written in the Slotboom formulation $[45,46]$ as

$$
\frac{\partial c}{\partial t}=\nabla \cdot(\bar{D} \nabla(\bar{c}))
$$

where

$$
\begin{aligned}
\bar{D} & =D e^{-\frac{V}{k_{B} T}} \\
\bar{c} & =c e^{\frac{V}{k_{B} T}}
\end{aligned}
$$

Following this transformation, the steady-state diffusion equation has the same form as the Fickian diffusion equation, with a spatially varying diffusion coefficient.

\subsubsection{Homogenized model of continuum and atomistic scale methane diffusion}

Homogenization $[11,12]$ is a procedure for separating scales in a partial differential equation for a periodic geometry. The equation used as an input to the procedure describes the processes that takes place at the smaller scale. The procedure produces an equation appropriate for analysis at the larger scale, which contains a parameter found by integrating over the unit cell at the smaller scale. For the homogenization approach to be valid, the relevant length scales must be well-separated. For notational clarity, we denote spatial coordinates at the larger scale by $x_{i}$ and at the smaller scale by $y_{i}$. The volume of the unit cell is denoted as $|Y|$.

Using the procedure described in Chapter 1 of Bensoussan, Lions, and Papanicolaou [12], the homogenization of Fick's law indicates that the diffusion matrix for the larger scale is:

$$
D_{i j}^{\text {macro }}=\frac{1}{|Y|}\left(\int_{Y}\left(D_{i j}-D_{i k} \frac{\partial \chi_{j}}{\partial y_{k}}\right) d^{n} y\right)
$$

where the vector $\chi$ is defined as the solution to

$$
\frac{\partial}{\partial y_{i}}\left(D_{i k} \frac{\partial \chi_{j}}{\partial y_{k}}\right)=\frac{\partial}{\partial y_{i}} D_{i j}
$$

Homogenization of the Smoluchowski equation was accomplished by using the Slotboom transformation, followed by using the homogenized Fickian equation with a spatially varying diffusion coefficient. In this case, the integral over the unit cell provides a value of $\bar{D}$ for the larger scale. For the inverse Slotboom transformation to obtain the Fickian diffusion coefficient, $D$, for the larger scale, the potential to be used is a constant value representing the potential of the surroundings at the larger scale. This potential must use the same gauge condition as the smaller-scale potential. 


\subsubsection{Flux-based calculation of effective diffusion coefficient}

For sufficiently pore simple geometries, such as the channel through the silicate, an effective diffusion coefficient can be obtained without the use of homogenization theory. This approach is based on solution of the steady-state diffusion equation, which is either the Fickian diffusion equation or the Smoluchowski equation herein. After the steady-state concentration field has been obtained from solution of the Partial Differential Equation (PDE), using the Smoluchowski definition of the flux:

$$
\vec{j}=-D e^{-\frac{V}{k_{B} T}} \vec{\nabla}\left(e^{\frac{V}{k_{B} T}} c\right)
$$

Integrating this flux over a cross-section of the pore gives a total flux, $J$. By continuity requirements the total flux must be the same at all cross-sections of the pore. From the perspective of a larger scale, this total flux must also satisfy a one-dimensional Fick's First Law using the effective diffusion coefficient for the silicate:

$$
J=-D_{\text {macro }} \frac{\Delta c}{\Delta x}
$$

where $\Delta c / \Delta x$ is the large-scale concentration gradient applied to the silicate. This gradient is obtained from the boundary conditions applied to the finite element model. Solving Equation 24 for $D_{\text {macro }}$ allows the effective diffusion coefficient to be determined from the integrated flux obtained from the simulation and the applied boundary conditions. While this approach is only applicable to simple geometries, the homogenization approach described in Sect. 6.2.2 can be applied to more complicated geometries.

\subsubsection{Computational details}

The finite element meshes for homogenization were created in GMSH [47]. The Finite Element Method (FEM) analysis was conducting with python code relying on version 2019.1.0 of the FEniCS package [48, 49].

In homogenization, the boundary conditions for the unit cell are that the corrector function should be periodic, with period matching the unit cell [12]. The python scripts using FEniCS were able to implement periodic boundary conditions for two-dimensional problems. While FEniCS supports periodic boundary conditions in three-dimensions, the programming effort required is considerably greater. Consequently, periodic boundary conditions were implemented for twodimensional unit cells only. For three-dimensional unit cells, Dirichlet boundary conditions were used instead, with the corrector set to zero at the boundaries. While this is technically a periodic condition, it may be too restrictive for the corrector functions in some cases. Generally, this boundary condition is appropriate for situations where barriers to diffusion within the unit cell are located away from the cell boundaries.

All code written in support of this publication is publicly available at https://github.com/huskeypm/pkhlab-analyses. Simulation input files and generated data are available upon request. 


\section{Supplement}

\section{S Acknowledgements}

Earlier results of this work were published in the doctoral dissertation of Tom

Pace. The authors gratefully acknowledge support by the Petroleum Research

Fund of the American Chemical Society. The authors are also grateful to Markus

Schmuck for helpful conversations about homogenization theory.

\section{References}

[1] J. Bear. Dynamics of fluids in porous media. Environmental science series (New York, 1972- ). New York: American Elsevier Pub. Co, 1972. ISBN: 978-0-444-00114-6.

[2] F. A. Coutelieris and J. M. P. Q. Delgado. Transport processes in porous media. en. Advanced structured materials v.20. OCLC: ocn769420091. Heidelberg ; New York: Springer, 2012. ISBN: 978-3-642-27909-6.

[3] S. Capponi et al. "Structural Relaxation Processes and Collective Dynamics of Water in Biomolecular Environments". en. In: The Journal of Physical Chemistry B 123.2 (Jan. 2019), pp. 480-486. ISSN: 1520-6106, 1520-5207.

[4] X. Cai et al. "Structure of water confined between two parallel graphene plates". en. In: The Journal of Chemical Physics 150.12 (Mar. 2019), p. 124703. ISSN: 0021-9606, 1089-7690.

[5] A. J. Rieth et al. "Hydrogen bonding structure of confined water templated by a metal-organic framework with open metal sites". en. In: Nature Communications 10.1 (Dec. 2019), p. 4771. ISSN: 2041-1723.

[6] N. Hansen et al. "Analysis of Diffusion Limitation in the Alkylation of Benzene over H-ZSM- 5 by Combining Quantum Chemical Calculations, Molecular Simulations, and a Continuum Approach". en. In: The Journal of Physical Chemistry C 113.1 (Jan. 2009), pp. 235-246. Issn: 1932-7447, 1932-7455.

[7] N. Hansen and F. J. Keil. "Multiscale Modeling of Reaction and Diffusion in Zeolites: From the Molecular Level to the Reactor". en. In: Soft Materials 10.1-3 (Jan. 2012), pp. 179-201. ISSN: 1539-445X, 1539-4468.

[8] T. Bui et al. "Transport Mechanism of Guest Methane in Water-Filled Nanopores". en. In: The Journal of Physical Chemistry C 121.29 (July 2017), pp. 15675-15686. ISSN: 1932-7447, 1932-7455.

[9] F. Sicard et al. "Position-Dependent Diffusion from Biased Simulations and Markov State Model Analysis". en. In: Journal of Chemical Theory and Computation 17.4 (Apr. 2021), pp. 2022-2033. ISSN: 1549-9618, 15499626 .

[10] M. Collin et al. "Molecular Dynamics Simulations of Water Structure and Diffusion in a $1 \mathrm{~nm}$ Diameter Silica Nanopore as a Function of Surface Charge and Alkali Metal Counterion Identity". en. In: The Journal of Physical Chemistry C 122.31 (Aug. 2018), pp. 17764-17776. ISSN: 19327447, 1932-7455.

[11] J.-L. Auriault, C. Boutin, and C. Geindreau. Homogenization of coupled phenomena in heterogenous media. eng. London, UK; Hoboken, N.J: ISTE; J. Wiley, 2009. ISBN: 978-1-84821-161-2. 
[12] A. Bensoussan, J.-L. Lions, and G. Papanicolaou. Asymptotic analysis for periodic structures. Providence, R.I: American Mathematical Society, 2011. ISBN: 978-0-8218-5324-5.

[13] Y. Ichikawa and A. P. S. Selvadurai. Transport Phenomena in Porous Media Aspects of Micro/Macro Behaviour. German. OCLC: 851089185. 2012. ISBN: 978-3-642-25333-1 978-3-642-25332-4.

[14] F. S. Emami et al. "Force Field and a Surface Model Database for Silica to Simulate Interfacial Properties in Atomic Resolution". en. In: Chemistry of Materials 26.8 (Apr. 2014), pp. 2647-2658. ISSN: 0897-4756, 1520-5002.

[15] F. S. Emami et al. "Correction to Force Field and a Surface Model Database for Silica to Simulate Interfacial Properties in Atomic Resolution". en. In: Chemistry of Materials 28.1 (Jan. 2016), pp. 406-407. ISSN: 0897-4756, 1520-5002.

[16] P. A. Witherspoon and D. N. Saraf. "Diffusion of Methane, Ethane, Propane, and n-Butane in Water from 25 to $43^{\circ}$ ". en. In: The Journal of Physical Chemistry 69.11 (Nov. 1965), pp. 3752-3755. ISSN: 0022-3654, 1541-5740.

[17] D. Frenkel and B. Smit. Understanding molecular simulation: from algorithms to applications. 2nd ed. Computational science series 1. San Diego: Academic Press, 2002. ISBN: 978-0-12-267351-1.

[18] W. L. Jorgensen, J. D. Madura, and C. J. Swenson. "Optimized intermolecular potential functions for liquid hydrocarbons". en. In: Journal of the American Chemical Society 106.22 (Oct. 1984), pp. 6638-6646. ISSN: 0002-7863.

[19] J. O. Daldrop, B. G. Kowalik, and R. R. Netz. "External Potential Modifies Friction of Molecular Solutes in Water". en. In: Physical Review X 7.4 (Dec. 2017). ISSN: 2160-3308.

[20] C. Oostenbrink et al. "A biomolecular force field based on the free enthalpy of hydration and solvation: The GROMOS force-field parameter sets 53A5 and 53A6". en. In: Journal of Computational Chemistry 25.13 (Oct. 2004), pp. 1656-1676. ISSN: 0192-8651, 1096-987X.

[21] P. M. Kekenes-Huskey et al. "Molecular and Subcellular-Scale Modeling of Nucleotide Diffusion in the Cardiac Myofilament Lattice". en. In: Biophysical Journal 105.9 (Nov. 2013), pp. 2130-2140. ISSN: 00063495.

[22] H. Rahmaninejad et al. "Co-localization and confinement of ecto-nucleotidases modulate extracellular adenosine nucleotide distributions": en. In: PLOS Computational Biology 16.6 (June 2020). Ed. by J. M. Briggs, e1007903. ISSN: $1553-7358$.

[23] G. Hummer. "Position-dependent diffusion coefficients and free energies from Bayesian analysis of equilibrium and replica molecular dynamics simulations". en. In: New Journal of Physics 7 (Feb. 2005), pp. 34-34. ISSN: 1367-2630.

[24] T. B. Woolf and B. Roux. "Conformational Flexibility of o-Phosphorylcholine and o-Phosphorylethanolamine: A Molecular Dynamics Study of Solvation Effects". en. In: Journal of the American Chemical Society 116.13 (June 1994), pp. 5916-5926. ISSN: 0002-7863.

[25] P. Setny et al. "Solvent fluctuations in hydrophobic cavity-ligand binding kinetics". en. In: Proceedings of the National Academy of Sciences 110.4 (Jan. 2013), pp. 1197-1202. ISSN: 0027-8424, 1091-6490. 
[26] R. Zwanzig. Nonequilibrium statistical mechanics. Oxford University Press, 2001.

[27] F. Reif. Fundamentals of statistical and thermal physics. eng. reiss. OCLC: 845506926. Long Grove, Ill: Waveland Press, 2009. ISBN: 978-1-57766-6127.

[28] R. K. Pathria and P. D. Beale. Statistical mechanics. 3rd ed. Amsterdam ; Boston: Elsevier/Academic Press, 2011. IsBN: 978-0-12-382188-1.

[29] S. K. Bhatia and D. Nicholson. "Adsorption and Diffusion of Methane in Silica Nanopores: A Comparison of Single-Site and Five-Site Models". en. In: The Journal of Physical Chemistry C 116.3 (Jan. 2012), pp. 23442355. ISSN: 1932-7447, 1932-7455.

[30] F. S. Emami et al. "Force Field and a Surface Model Database for Silica to Simulate Interfacial Properties in Atomic Resolution". In: Chemistry of Materials 26.8 (2014), pp. 2647-2658.

[31] W. L. Jorgensen, J. D. Madura, and C. J. Swenson. "Optimized intermolecular potential functions for liquid hydrocarbons". In: Journal of the American Chemical Society 106.22 (1984), pp. 6638-6646.

[32] T. Darden, D. York, and L. Pedersen. "Particle mesh Ewald: An $N \log ($ $N$ ) method for Ewald sums in large systems". en. In: The Journal of Chemical Physics 98.12 (June 1993), pp. 10089-10092. ISSN: 0021-9606, 1089-7690.

[33] U. Essmann et al. "A smooth particle mesh Ewald method". en. In: The Journal of Chemical Physics 103.19 (Nov. 1995), pp. 8577-8593. ISSN: 0021-9606, 1089-7690.

[34] B. Hess, H. Bekker, and H. J. C. Berendsen. "LINCS: A linear constraint solver for molecular simulations". en. In: JOURNAL OF COMPUTATIONAL CHEMISTRY 18.12 (1997), p. 10.

[35] R. Gowers et al. "MDAnalysis: A Python Package for the Rapid Analysis of Molecular Dynamics Simulations". In: Proceedings of the 15th Python in Science Conference. SciPy, 2016.

[36] N. Michaud-Agrawal et al. "MDAnalysis: A toolkit for the analysis of molecular dynamics simulations". In: Journal of Computational Chemistry 32.10 (Apr. 2011). Publisher: Wiley, pp. 2319-2327.

[37] S. Van Der Walt, S. C. Colbert, and G. Varoquaux. "The NumPy array: a structure for efficient numerical computation". In: Computing in Science E Engineering 13.2 (2011). Publisher: AIP Publishing, pp. 22-30.

[38] A. Grossfield. WHAM: an implementation of the weighted histogram analysis method, Version 2.0.10. 2005.

[39] S. Kumar et al. "THE weighted histogram analysis method for free-energy calculations on biomolecules. I. The method". en. In: Journal of Computational Chemistry 13.8 (Oct. 1992), pp. 1011-1021. ISSN: 0192-8651, 1096-987X.

[40] S. Kumar et al. "Multidimensional free-energy calculations using the weighted histogram analysis method". en. In: Journal of Computational Chemistry 16.11 (Nov. 1995), pp. 1339-1350. ISSN: 0192-8651, 1096-987X.

[41] B. Roux. "The calculation of the potential of mean force using computer simulations". en. In: Computer Physics Communications 91.1-3 (Sept. 1995), pp. 275-282. ISSN: 00104655. 
[42] J. Crank. Mathematics of Diffusion. Oxford: Clarendon Press, 1970.

[43] M. S. Miguel and J. M. Sancho. "A colored-noise approach to Brownian motion in position space. Corrections to the Smoluchowski equation". en. In: Journal of Statistical Physics 22.5 (May 1980), pp. 605-624. ISSN: 0022-4715, 1572-9613.

[44] K. Schulten and I. Kosztin. "Lectures in Theoretical Biophysics". University of Illinois at Urbana-Champaign, 2000.

[45] B. Lu et al. "Poisson-Nernst-Planck equations for simulating biomolecular diffusion-reaction processes I: Finite element solutions". en. In: Journal of Computational Physics 229.19 (Sept. 2010), pp. 6979-6994. ISSN: 00219991.

[46] J. Slotboom. "Computer-aided two-dimensional analysis of bipolar transistors". en. In: IEEE Transactions on Electron Devices 20.8 (Aug. 1973), pp. 669-679. ISSN: 0018-9383.

[47] C. Geuzaine and J.-F. Remacle. "Gmsh: A 3-D finite element mesh generator with built-in pre- and post-processing facilities: THE GMSH PAPER". en. In: International Journal for Numerical Methods in Engineering 79.11 (Sept. 2009), pp. 1309-1331. ISSN: 00295981.

[48] M. S. Alnæs et al. "The FEniCS Project Version 1.5". In: Archive of Numerical Software 3.100 (2015).

[49] A. Logg, G. N. Wells, and J. Hake. "DOLFIN: a C++/Python Finite Element Library". In: Automated Solution of Differential Equations by the Finite Element Method, Volume 84 of Lecture Notes in Computational Science and Engineering. Ed. by A. Logg, K.-A. Mardal, and G. N. Wells. Springer, 2012. Chap. 10. 


\section{Supplementary Information (SI)}

\section{S1 Supplementary Tables}

Table S1: MSD results from simulations of unrestrained methane in bulk water. The theoretical result is based on the simulation temperature and the applied harmonic potential.

\begin{tabular}{cccc} 
Direction & $\begin{array}{c}\text { Theoretical } \\
\text { Result } \\
\left(\mathrm{nm}^{2}\right)\end{array}$ & $\begin{array}{c}\text { Jorgensen/SPC } \\
\text { Force Field } \\
\left(\mathrm{nm}^{2}\right)\end{array}$ & $\begin{array}{c}\text { GROMOS 53A6 } \\
\text { Force Field } \\
\left(\mathrm{nm}^{2}\right)\end{array}$ \\
\hline $\mathrm{x}$ & 0.0083 & 0.0081 & 0.0081 \\
$\mathrm{y}$ & 0.0083 & 0.0079 & 0.0081 \\
$\mathrm{z}$ & 0.0083 & 0.0083 & 0.0079
\end{tabular}

\section{S2 Supplementary Figures}

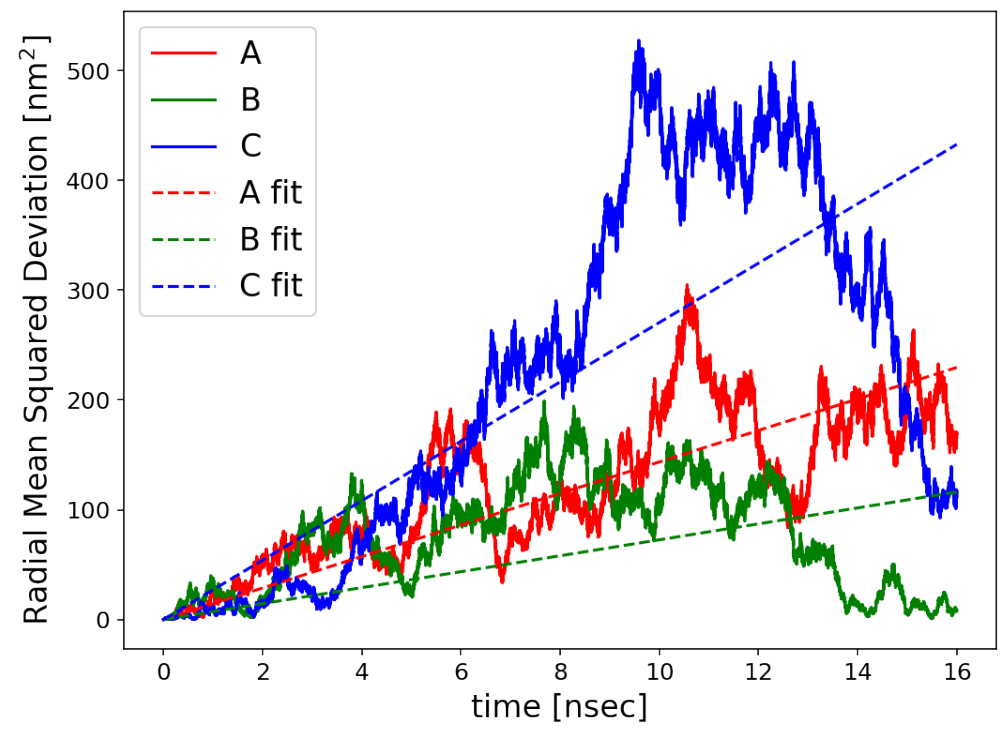

Figure S1: Calculation of the diffusion coefficient from the MSD of unrestrained methane, for three different trials. Each simulation had a duration of $16 \mathrm{~ns}$. 

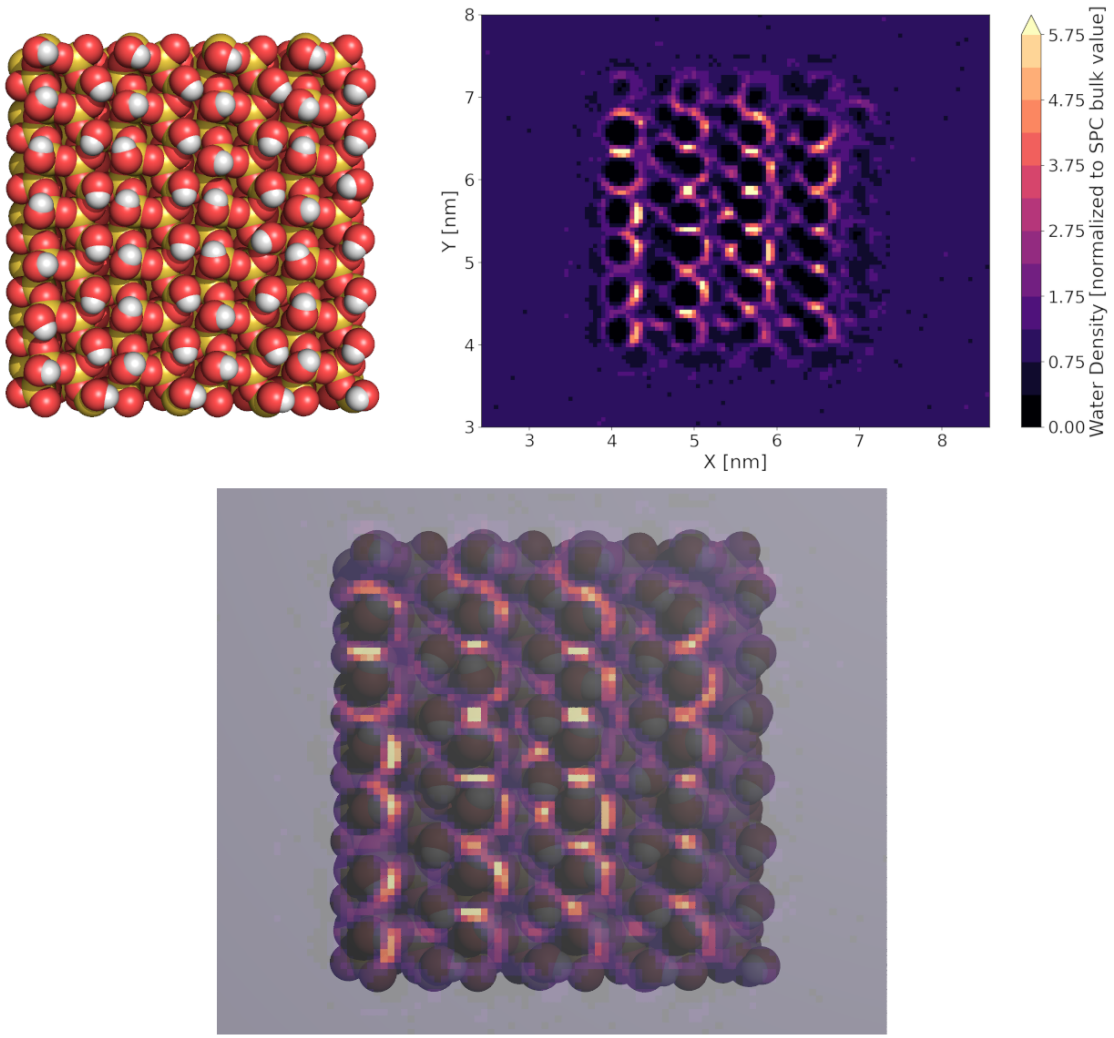

Figure S2: Water density near the silicate face from the MD simulation with a channel width of $1.6 \mathrm{~nm}$. Top left: rendering of the silicate face. Top right: water density results for the plane at $z=4.775 \mathrm{~nm}$, which is approximately where the water density values reach a local maximum in some areas. Bottom: scaled overlay of the silicate structure and the water density data. The water molecule positions are referenced to the center of their oxygen atoms, so the density is highest around the silanol terminations of the silicate surface. The water density was calculated at a spatial resolution of $0.5 \AA$, from 128 nanoseconds of simulation data. 


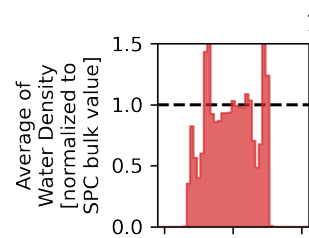

$1.2 \mathrm{~nm}$ width, $100 \%$ protonation
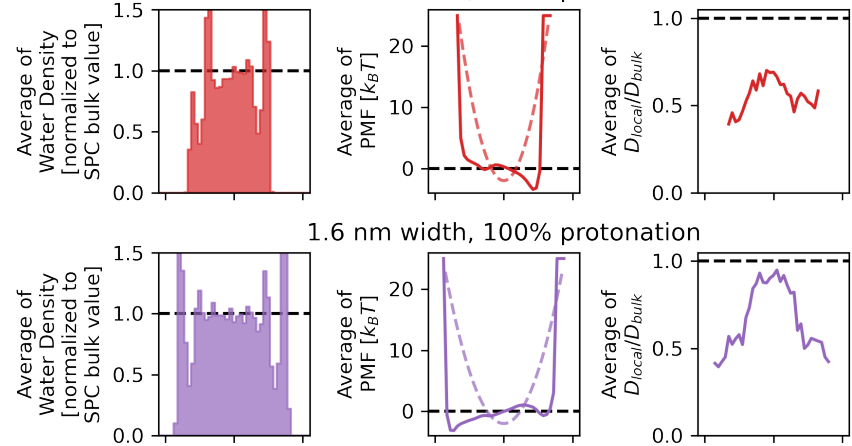

$1.6 \mathrm{~nm}$ width, $100 \%$ protonation
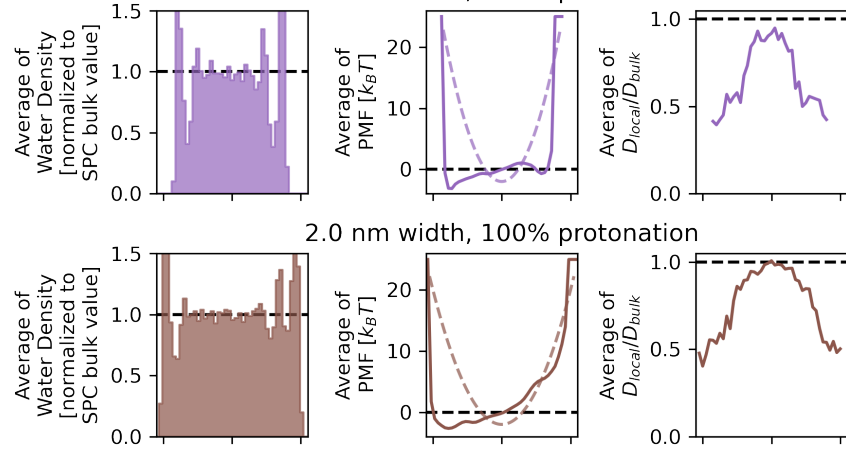

$2.0 \mathrm{~nm}$ width, $100 \%$ protonation
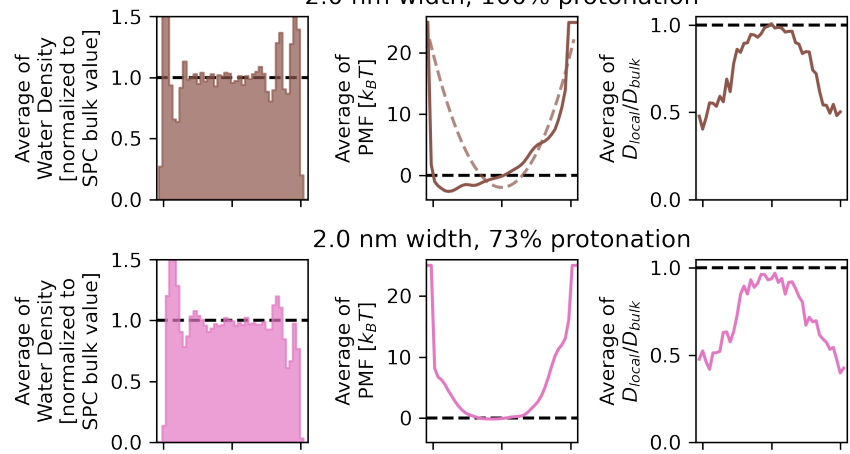

$.0 \mathrm{~nm}$ width, $73 \%$ protonation
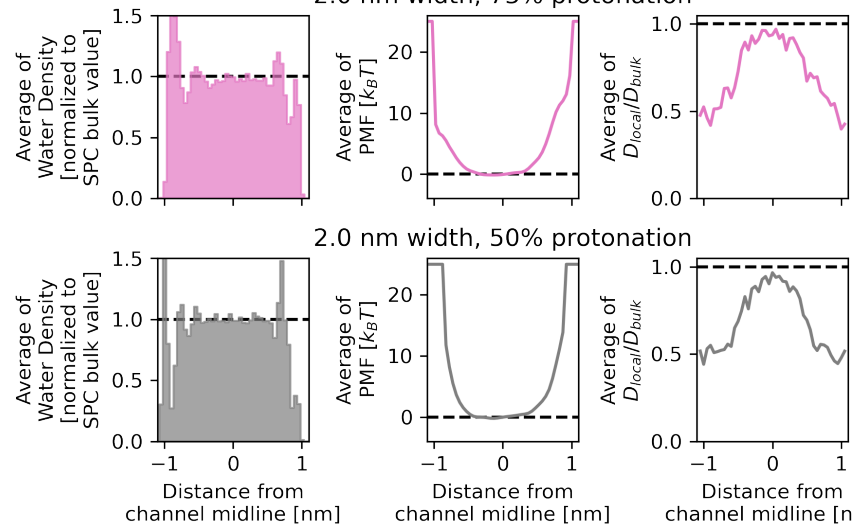

$2.0 \mathrm{~nm}$ width, $50 \%$ protonation
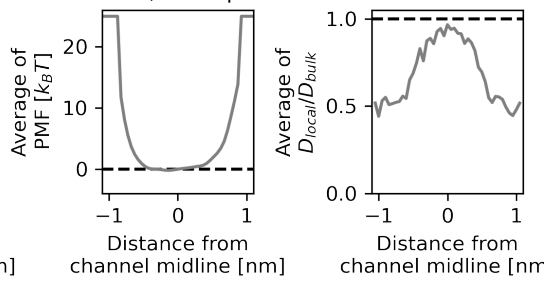

Figure S3: Spatial variation of water density, Potential of Mean Force (PMF), and local diffusion coefficients for all five combinations of channel width and protonation level investigated in the molecular dynamics (MD) simulations. These results are averaged over the $y$ direction to show variation across the channel. Bulk values are shown as black dashed lines. The quadratic potentials used for the validation described in Sect. 3.2 are shown on the PMF plots where applicable, as dashed lines with the same color as the PMF data from MD. 


\section{S3 Analytical Solution for a Quadratic Potential}

\section{S3.1 Problem description}

The problem is defined in two spatial dimensions. The unit cell is shown in Figure S4. Two scales of analysis are present: $y$ for the smaller scale, with characteristic length matching the size of the unit cell, and $x$ for the larger scale.

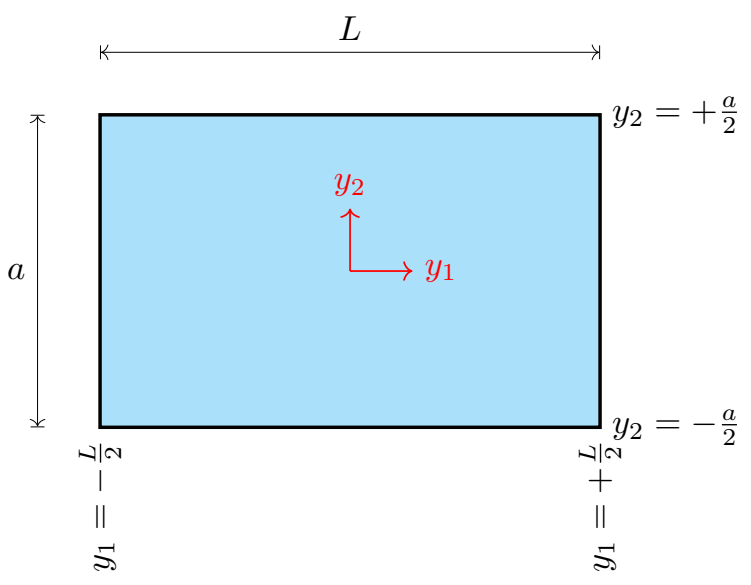

Figure S4: Unit cell geometry.

Within the unit cell, there is a quadratic potential of mean force:

$$
\psi=\psi(y)=A y_{2}^{2}+B
$$

where $A$ and $B$ are scalar constants. Note that this potential is periodic, but its gradient is not:

$$
\begin{aligned}
\frac{\partial}{\partial y_{1}} \psi & =0 \\
\frac{\partial}{\partial y_{2}} \psi & =2 A y_{2}
\end{aligned}
$$

Particles are allowed to diffuse through the unit cell subject to the Smoluchowsi equation:

$$
j_{i}=-D\left(\frac{\partial}{\partial y_{i}} c+\beta c \frac{\partial}{\partial y_{i}} \psi\right)
$$

where

$c=c(x, y)$, the concentration

$j_{i}=j_{i}(x, y)$, the flux vector

$D=$ isotropic and spatially invariant local diffusion coefficient for the smaller scale

$\beta=$ inverse of $k_{B} T$, spatially invariant

The Smoluchowski equation can be written in the following equivalent form, with the equivalence easily verified by applying the product rule for the gradient operator:

$$
j_{i}=-D e^{-\beta \psi} \frac{\partial}{\partial y_{i}}\left(c e^{\beta \psi}\right)
$$


This form allows the application of the Slotboom transformation:

$$
\begin{aligned}
\bar{D} & =D e^{-\beta \psi} \\
\bar{c} & =c e^{\beta \psi}
\end{aligned}
$$

With this transformation, the Smoluchowski equation simply becomes:

$$
j_{i}=-\bar{D} \frac{\partial}{\partial y_{i}} \bar{c}
$$

For the quadratic potential described above,

$$
\begin{aligned}
& \bar{D}=D e^{-\beta A y_{2}^{2}} e^{-\beta B} \\
& \frac{\partial}{\partial y_{1}} \bar{D}=0 \\
& \frac{\partial}{\partial y_{2}} \bar{D}=-2 \beta A y_{2} \bar{D}
\end{aligned}
$$

In addition to the Smoluchowski equation, continuity is also satisfied, and steady-state conditions are assumed. These two conditions together impose the requirement:

$$
\frac{\partial}{\partial y_{i}} j_{i}=0
$$

(Summation is implied by the repeated index.) Combining this with the Smoluchowski flux:

$$
\frac{\partial}{\partial y_{i}}\left(\bar{D} \frac{\partial}{\partial y_{i}} \bar{c}\right)=0
$$

The flux for the larger scale is defined by the averages of the smaller-scale flux:

$$
\begin{aligned}
& J_{1}=\frac{1}{a} \int_{-\frac{a}{2}}^{\frac{a}{2}} d y_{2} j_{1} \\
& J_{2}=\frac{1}{L} \int_{-\frac{L}{2}}^{\frac{L}{2}} d y_{1} j_{2}
\end{aligned}
$$

Finally, it is necessary to define the value of the potential at the larger scale. This is a static constant, and must use the same gauge condition as the smallscale potential.

$$
\Psi=\text { potential value at the large scale, a constant }
$$

The objective is to obtain the effective diffusion coefficient, $\widehat{D}$, for the larger scale, for Fickian diffusion:

$$
J_{i}=-\widehat{D}_{i j} \frac{\partial}{\partial x_{j}} c
$$

Both a flux-based calculation and homogenization analysis will be used to derive the same analytical result.

\section{S3.2 Flux-based calculation}

\section{S3.2.1 General approach}

The components of $\widehat{D}_{i j}$ can be found by imposing two different concentration gradients on the unit cell. These two different concentration gradients are orthogonal to one another, and imposed separately.

First, a concentration difference, $\Delta_{1} c$, is applied between the left and right edges of the unit cell. No gradient is applied in the orthogonal direction. The 
upper-scale gradient is approximated as:

$$
\frac{\partial}{\partial x_{1}} c \approx \frac{\Delta_{1} c}{L}
$$

The Fickian diffusion equation at the upper scale for this situation expands as:

$$
\left[\begin{array}{l}
J_{1} \\
J_{2}
\end{array}\right]=-\left[\begin{array}{ll}
\widehat{D}_{11} & \widehat{D}_{12} \\
\widehat{D}_{21} & \widehat{D}_{22}
\end{array}\right]\left[\begin{array}{l}
\frac{\Delta_{1} c}{f} \\
0
\end{array}\right]
$$

This is equivalent to:

$$
\begin{aligned}
& J_{1}=-\widehat{D}_{11} \frac{\Delta_{1} c}{L} \\
& J_{2}=-\widehat{D}_{21} \frac{\Delta_{1} c}{L}
\end{aligned}
$$

These expressions can be re-arranged to obtain two components of the effective diffusion coefficient:

$$
\begin{aligned}
& \widehat{D}_{11}=-J_{1} \frac{L}{\Delta_{1} c} \\
& \widehat{D}_{21}=-J_{2} \frac{L}{\Delta_{1} c}
\end{aligned}
$$

Thus, if a solution for $c$ can be obtained for the boundary value problem, the fluxes $j_{1}$ and $j_{2}$ can be computed. Then the fluxes $J_{1}$ and $J_{2}$ can obtained from integration, and then $\widehat{D}_{11}$ and $\widehat{D}_{21}$ can be obtained as well.

Similarly, a concentration difference $\Delta_{2} c$ is imposed between the upper and lower boundaries of the unit cell:

$$
\frac{\partial}{\partial x_{2}} c \approx \frac{\Delta_{2} c}{a}
$$

And so,

$$
\left[\begin{array}{l}
J_{1} \\
J_{2}
\end{array}\right]=-\left[\begin{array}{ll}
\widehat{D}_{11} & \widehat{D}_{12} \\
\widehat{D}_{21} & \widehat{D}_{22}
\end{array}\right]\left[\begin{array}{c}
0 \\
\frac{\Delta_{2} c}{a}
\end{array}\right]
$$

This is equivalent to:

$$
\begin{aligned}
& J_{1}=-\widehat{D}_{11} \frac{\Delta_{2} c}{a} \\
& J_{2}=-\widehat{D}_{22} \frac{\Delta_{2} c}{a}
\end{aligned}
$$

Re-arranging:

$$
\begin{aligned}
& \widehat{D}_{12}=-J_{1} \frac{a}{\Delta_{2} c} \\
& \widehat{D}_{22}=-J_{2} \frac{a}{\Delta_{2} c}
\end{aligned}
$$

And so, by imposing two separate concentration boundary conditions on the unit cell, all four components of $\widehat{D}_{i j}$ can be obtained.

\section{S3.2.2 Imposed gradient from left to right}

To satisfy the steady-state condition, the concentration at the boundary must be related to the potential by a Poisson-Boltzmann distribution. This can be achieved by setting a constant value of $\bar{c}$. To impose a left-right concentration gradient, the boundary conditions are:

$$
\begin{aligned}
& \bar{c}\left(y_{1}=-\frac{L}{2}\right)=\bar{c}_{L} \\
& \bar{c}\left(y_{1}=+\frac{L}{2}\right)=\bar{c}_{R}
\end{aligned}
$$


For these boundary conditions, the governing PDE (Equation S10) is solved by:

$$
\bar{c}\left(y_{1}, y_{2}\right)=\bar{c}\left(y_{1}\right)=\frac{\bar{c}_{R}-\bar{c}_{L}}{L} y_{1}+\frac{\bar{c}_{R}+\bar{c}_{L}}{2}
$$

The fluxes resulting from this solution are:

$$
\begin{aligned}
& j_{1}=-\bar{D} \frac{\bar{c}_{R}-\bar{c}_{L}}{L} \\
& j_{2}=0
\end{aligned}
$$

The larger-scale fluxes are:

$$
\begin{gathered}
J_{1}=\frac{1}{a} \int_{-\frac{a}{2}}^{\frac{a}{2}} d y_{2} j_{1}=-D \frac{\bar{c}_{R}-\bar{c}_{L}}{a L} \int_{-\frac{a}{2}}^{\frac{a}{2}} d y_{2} e^{-\beta \psi\left(y_{2}\right)} \\
J_{2}=\frac{1}{L} \int_{-\frac{L}{2}}^{\frac{L}{2}} d y_{1} j_{2}=0
\end{gathered}
$$

Before components of $\widehat{D}$ can be obtained by Equation $\mathrm{S} 17$, the value of $\Delta_{1} c$ must be defined. Because of the Poisson-Boltzmann distribution, the concentration varies along the left and right boundaries. The large-scale concentration at the boundary is computed from $\bar{c}$ at the boundary and the constant value of the potential at the large scale.

$$
\Delta_{1} c=\left(\bar{c}_{R}-\bar{c}_{L}\right) e^{-\beta \Psi}
$$

And so, from Equation S17,

$$
\begin{gathered}
\widehat{D}_{11}=D e^{+\beta \Psi} \frac{1}{a} e^{-\beta B} \int_{-\frac{a}{2}}^{\frac{a}{2}} d y_{2} e^{-\beta A y_{2}^{2}} \\
\widehat{D}_{21}=0
\end{gathered}
$$

The influence of the potential on the effective diffusion coefficient reflects the change in the potential between the unit cell and the surroundings at the large scale.

\section{S3.2.3 Imposed gradient from bottom to top}

To impose a vertical concentration gradient, the boundary conditions are:

$$
\begin{aligned}
& \bar{c}\left(y_{2}=-\frac{a}{2}\right)=\bar{c}_{B} \\
& \bar{c}\left(y_{2}=+\frac{a}{2}\right)=\bar{c}_{T}
\end{aligned}
$$

For these boundary conditions, the governing PDE (Equation S10) is solved by:

$$
\bar{c}\left(y_{1}, y_{2}\right)=\bar{c}\left(y_{2}\right)=K \int_{0}^{y_{2}} d s e^{\beta \psi(s)}+k
$$

where $K$ and $k$ are constants depending on the boundary conditions. The value of $k$ will not be relevant for the flux calculations. For the given boundary conditions, $K$ is:

$$
K=\frac{\bar{c}_{T}-\bar{c}_{B}}{\int_{-\frac{a}{2}}^{\frac{a}{2}} d y_{2} e^{\beta \psi\left(y_{2}\right)}}=\frac{\bar{c}_{T}-\bar{c}_{B}}{e^{\beta B} \int_{-\frac{a}{2}}^{\frac{a}{2}} d y_{2} e^{\beta A y_{2}^{2}}}
$$


The fluxes resulting from this solution are:

$$
\begin{aligned}
& j_{1}=0 \\
& j_{2}=-\bar{D} K e^{\beta \psi\left(y_{2}\right)}=-D K
\end{aligned}
$$

The larger-scale fluxes are:

$$
\begin{aligned}
& J_{1}=0 \\
& J_{2}=-D K
\end{aligned}
$$

The larger-scale concentration difference is

$$
\Delta_{2} c=\left(\bar{c}_{T}-\bar{c}_{B}\right) e^{-\beta \Psi}
$$

to be consistent with the steady-state solution of the Smoluchowski equation at the larger scale. And so, from Equation S21,

$$
\begin{gathered}
\widehat{D}_{12}=0 \\
\widehat{D}_{22}=D e^{+\beta \Psi} \frac{a}{e^{\beta B} \int_{-\frac{a}{2}}^{\frac{a}{2}} d y_{2} e^{\beta A y_{2}^{2}}}
\end{gathered}
$$

Even though the result now depends on both $A$ and $B$, it is still gaugeinvariant, because a gauge shift in the value of $B$ would be offset by a gauge shift in the value of $\Psi$.

\section{S3.2.4 Summary}

Combining the results of both flux calculations, the entire matrix for $\widehat{D}$ has been obtained.

$$
\widehat{D}_{i j}=D e^{+\beta \Psi}\left[\begin{array}{cc}
\frac{1}{a} \int_{-\frac{a}{2}}^{\frac{a}{2}} d y_{2} e^{-\beta \psi\left(y_{2}\right)} & 0 \\
0 & \frac{1}{\frac{1}{a} \int_{-\frac{a}{2}}^{\frac{a}{2}} d y_{2} e^{+\beta \psi\left(y_{2}\right)}}
\end{array}\right]
$$

This result is consistent with Fickian diffusion in layered media: the results in one direction are the average of the layer properties, and in the other direction are the inverse of the average of the inverses. Here, the sums over individual layers are replaced with integrals over a single dimension.

As noted above, the results are gauge-independent so long as the large-scale potential $\Psi$ and the small-scale potential $\psi$ use the same gauge condition.

\section{S3.3 Homogenization analysis}

\section{S3.3.1 Corrector problem}

As the problem is two-dimensional, there are two components of the corrector function, $\chi$, to be found. For isotropic Fickian diffusion, the corrector problem is:

$$
\frac{\partial}{\partial y_{i}}\left(D \frac{\partial}{\partial y_{i}} \chi_{j}\right)=\frac{\partial}{\partial y_{j}} D
$$


For the Slotboom-transformed Smoluchowski equation, this becomes

$$
\frac{\partial}{\partial y_{i}}\left(\bar{D} \frac{\partial}{\partial y_{i}} \chi_{j}\right)=\frac{\partial}{\partial y_{j}} \bar{D}
$$

For the quadratic potential described above, this expands to

$$
\frac{\partial}{\partial y_{i}} \frac{\partial}{\partial y_{i}} \chi_{j}-2 \beta A y_{2} \frac{\partial}{\partial y_{2}} \chi_{j}=-2 \beta A y_{2} \delta_{j 2}
$$

The two components of $\chi$ are therefore defined by the following PDEs:

$$
\frac{\frac{\partial^{2}}{\partial y_{1}^{2}} \chi_{1}+\frac{\partial^{2}}{\partial y_{2}^{2}} \chi_{1}-2 \beta A y_{2} \frac{\partial}{\partial y_{2}} \chi_{1}=0}{\frac{\partial^{2}}{\partial y_{1}^{2}} \chi_{2}+\frac{\partial^{2}}{\partial y_{2}^{2}} \chi_{2}-2 \beta A y_{2} \frac{\partial}{\partial y_{2}} \chi_{2}=-2 \beta A y_{2}}
$$

These problems only define the corrector functions up to the addition of an arbitrary constant. The partial derivatives of the corrector functions are the only results required for the unit cell integrals.

\section{S3.3.2 Solution for $\chi_{1}$}

The solution for $\chi_{1}$ is of the form

$$
\chi_{1}=Q \int d y_{2} e^{\beta A y_{2}^{2}}
$$

where $Q$ is a scalar constant determined by the requirement that $\chi_{1}$ be periodic. Specifically,

$$
\chi_{1}\left(y_{2}=-\frac{a}{2}\right)=\chi_{1}\left(y_{2}=\frac{a}{2}\right)
$$

This may be expressed as

$$
\begin{gathered}
Q \int_{0}^{-\frac{a}{2}} d y_{2} e^{\beta A y_{2}^{2}}=Q \int_{0}^{\frac{a}{2}} d y_{2} e^{\beta A y_{2}^{2}} \\
Q \int_{-\frac{a}{2}}^{\frac{a}{2}} d y_{2} e^{\beta A y_{2}^{2}}=0
\end{gathered}
$$

which requires $Q=0$. Consequently,

$$
\begin{aligned}
& \frac{\partial}{\partial y_{1}} \chi_{1}=0 \\
& \frac{\partial}{\partial y_{2}} \chi_{1}=0
\end{aligned}
$$

\section{S3.3.3 Solution for $\chi_{2}$}

The solution for $\chi_{2}$ is of the form

$$
\chi_{2}=y_{2}-G \int d y_{2} e^{\beta A y_{2}^{2}}
$$


where $G$ is a scalar constant determined by the requirement that $\chi_{2}$ be periodic. Specifically,

$$
\chi_{2}\left(y_{2}=-\frac{a}{2}\right)=\chi_{2}\left(y_{2}=\frac{a}{2}\right)
$$

This may be expressed as

$$
-\frac{a}{2}-G \int_{0}^{-\frac{a}{2}} d y_{2} e^{\beta A y_{2}^{2}}=\frac{a}{2}-G \int_{0}^{\frac{a}{2}} d y_{2} e^{\beta A y_{2}^{2}}
$$

which reduces to

$$
G=\frac{a}{\int_{-\frac{a}{2}}^{\frac{a}{2}} d y_{2} e^{\beta A y_{2}^{2}}}
$$

And so the partial derivatives are

$$
\begin{aligned}
\frac{\partial}{\partial y_{1}} \chi_{2} & =0 \\
\frac{\partial}{\partial y_{2}} \chi_{2} & =1-G e^{\beta A y_{2}^{2}}
\end{aligned}
$$

\section{S3.3.4 Unit cell integrals}

For isotropic Fickian diffusion, the effective diffusion coefficient is given by the following integral over the unit cell:

$$
\widehat{D}_{i j}=\frac{1}{|Y|} \int_{Y} d Y D\left(\delta_{i j}-\frac{\partial}{\partial y_{i}} \chi_{j}\right)
$$

For the Slotboom-transformed Smoluchowski equation, this becomes

$$
\widehat{\bar{D}}_{i j}=\frac{1}{|Y|} \int_{Y} d Y \bar{D}\left(\delta_{i j}-\frac{\partial}{\partial y_{i}} \chi_{j}\right)
$$

For the unit cell in question, this is

$$
\widehat{\bar{D}}_{i j}=D e^{-\beta B} \frac{1}{a L} \int_{-\frac{a}{2}}^{\frac{a}{2}} d y_{2} e^{-\beta A y_{2}^{2}} \int_{-\frac{L}{2}}^{\frac{L}{2}} d y_{1}\left(\delta_{i j}-\frac{\partial}{\partial y_{i}} \chi_{j}\right)
$$

This integral is evaluated to find all four components of $\widehat{\bar{D}}$.

$$
\begin{gathered}
\widehat{\bar{D}}_{11}=D e^{-\beta B} \frac{1}{a L} \int_{-\frac{a}{2}}^{\frac{a}{2}} d y_{2} e^{-\beta A y_{2}^{2}} \int_{-\frac{L}{2}}^{\frac{L}{2}} d y_{1}\left(1-\frac{\partial}{\partial y_{1}} \chi_{1}\right)=D e^{-\beta B} \frac{1}{a} \int_{-\frac{a}{2}}^{\frac{a}{2}} d y_{2} e^{-\beta A y_{2}^{2}} \\
\widehat{\bar{D}}_{12}=D e^{-\beta B} \frac{1}{a L} \int_{-\frac{a}{2}}^{\frac{a}{2}} d y_{2} e^{-\beta A y_{2}^{2}} \int_{-\frac{L}{2}}^{\frac{L}{2}} d y_{1}\left(0-\frac{\partial}{\partial y_{1}} \chi_{2}\right)=0 \\
\widehat{D}_{21}=D e^{-\beta B} \frac{1}{a L} \int_{-\frac{a}{2}}^{\frac{a}{2}} d y_{2} e^{-\beta A y_{2}^{2}} \int_{-\frac{L}{2}}^{\frac{L}{2}} d y_{1}\left(0-\frac{\partial}{\partial y_{2}} \chi_{1}\right)=0
\end{gathered}
$$




$$
\widehat{\bar{D}}_{22}=D e^{-\beta B} \frac{1}{a L} \int_{-\frac{a}{2}}^{\frac{a}{2}} d y_{2} e^{-\beta A y_{2}^{2}} \int_{-\frac{L}{2}}^{\frac{L}{2}} d y_{1}\left(1-\frac{\partial}{\partial y_{2}} \chi_{2}\right)=D G e^{-\beta B}=D \frac{a e^{-\beta B}}{\int_{-\frac{a}{2}}^{\frac{a}{2}} d y_{2} e^{\beta A y_{2}^{2}}}
$$

\section{S3.3.5 Summary}

From the unit cell integrals above, the matrix for $\widehat{\bar{D}}$ has been obtained.

$$
\widehat{D}_{i j}=D e^{-\beta B}\left[\begin{array}{cc}
\frac{1}{a} \int_{-\frac{a}{2}}^{\frac{a}{2}} d y_{2} e^{-\beta A y_{2}^{2}} & 0 \\
0 & \frac{a}{\int_{-\frac{a}{2}}^{\frac{a}{2}} d y_{2} e^{\beta A y_{2}^{2}}}
\end{array}\right]
$$

\section{S3.4 Conclusion}

Comparing the results above for the components of $\widehat{D}$ and $\widehat{D}$ :

$$
\begin{aligned}
& \widehat{D}_{11}=\widehat{\bar{D}}_{11} e^{+\beta \Psi} \\
& \widehat{D}_{22}=\widehat{\widehat{D}}_{22} e^{+\beta \Psi}
\end{aligned}
$$

In fact, this is exactly the result needed for consistency with the Slotboomtransformed Smoluchowski equation at the larger scale:

$$
J_{i}=-\widehat{\bar{D}}_{i j} \frac{\partial}{\partial x_{j}} \bar{c}
$$

Expanding the Slotboom-transformed concentration, this becomes:

$$
J_{i}=-\widehat{\bar{D}}_{i j} \frac{\partial}{\partial x_{j}} c e^{+\beta \Psi}
$$

And $\Psi$ is invariant at the larger scale, so this is also:

$$
J_{i}=-\widehat{\bar{D}}_{i j} e^{+\beta \Psi} \frac{\partial}{\partial x_{j}} c
$$

Comparing this to the Fickian diffusion equation at the larger scale,

$$
J_{i}=-\widehat{D}_{i j} \frac{\partial}{\partial x_{j}} c
$$

it is clear that the solution for $\widehat{D}$ is

$$
\widehat{D}=\widehat{\bar{D}} e^{+\beta \Psi}
$$

exactly as noted above for the nonzero components.

Finally, note that in a situation where the gauge condition is such that $\Psi=0$, this result implies that $\widehat{D}=\widehat{\bar{D}}$. 